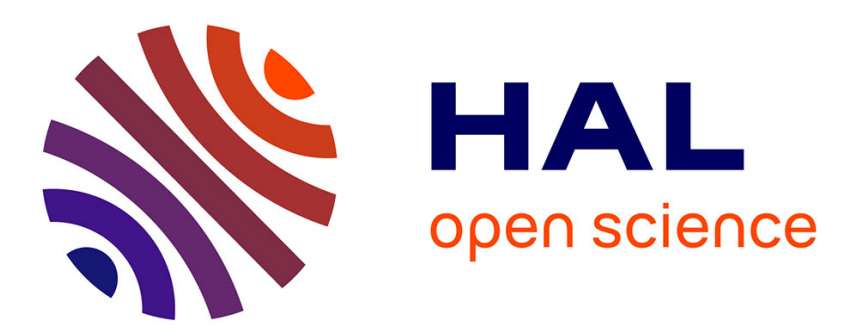

\title{
Decision making policy for RF energy harvesting enabled cognitive radios in decentralized wireless networks
}

Sumit J. Darak, Honggang Zhang, Jacques Palicot, Christophe Moy

\section{To cite this version:}

Sumit J. Darak, Honggang Zhang, Jacques Palicot, Christophe Moy. Decision making policy for RF energy harvesting enabled cognitive radios in decentralized wireless networks. Digital Signal Processing, 2017, 60, pp.33-45. 10.1016/j.dsp.2016.08.014 . hal-01436769

HAL Id: hal-01436769

https://hal-univ-rennes1.archives-ouvertes.fr/hal-01436769

Submitted on 18 May 2017

HAL is a multi-disciplinary open access archive for the deposit and dissemination of scientific research documents, whether they are published or not. The documents may come from teaching and research institutions in France or abroad, or from public or private research centers.
L'archive ouverte pluridisciplinaire HAL, est destinée au dépôt et à la diffusion de documents scientifiques de niveau recherche, publiés ou non, émanant des établissements d'enseignement et de recherche français ou étrangers, des laboratoires publics ou privés. 


\section{Highlights}

- Decision making policy for RF Energy Harvesting enabled Cognitive Radio.

- Thompson Sampling algorithm based subband statistic estimation and subband access.

- Proposed USRP testbed to validate the functionality of the various DMPs. 


\title{
Decision Making Policy for RF Energy Harvesting Enabled Cognitive Radios in Decentralized Wireless Networks
}

\author{
Sumit J. Darak ${ }^{\mathrm{a}, *}$, Honggang Zhang ${ }^{\mathrm{b}}$, Jacques Palicot ${ }^{\mathrm{c}}$, Christophe Moy ${ }^{\mathrm{c}}$ \\ ${ }^{a}$ Department of Electronics and Communications Engineering, IIIT-Delhi, India \\ ${ }^{b}$ Department of Information Science and Electronic Engineering (ISEE), Zhejiang \\ University, China \\ ${ }^{c}$ Department of Signal, Communication et Electronique Embarquée (SCEE), \\ CentraleSupélec/IETR, Rennes, France
}

\begin{abstract}
Cognitive radio (CR) paradigm with radio frequency energy harvesting (RFEH) have significant potential to improve the network throughput by utilizing vacant spectrum using battery-operated self-sustainable radio terminals. Research efforts relevant to these paradigms are focused on the mode selection policies which decide when to switch from $\mathrm{CR}$ mode (i.e., opportunistic vacant spectrum access mode) to the RFEH mode (i.e., battery charging using ambient RF energy) and vice-versa. So far, very little attention has been paid to the dual but competing task of frequency band selection in CR and RFEH modes under partially observable environment in the decentralized wireless networks. Furthermore, the need of tunable bandwidth frequency band access for CRs and lower subband switching cost (SSC) for energy efficient implementation have made the design of the decision making policy (DMP) more challenging. In this paper, a new CR-RFEH DMP has been proposed for RFEH enabled CR terminals in the decentralized wireless networks. The proposed DMP consists of three sub-units: 1) Bayesian approach based tunable Thompson sampling algorithm for subband statistics estimation, 2) Thompson sampling algorithm based subband access scheme exploiting the past collision events to minimize collisions among CRs, and 3) Mode selection scheme. Simulation results show that the proposed DMP offers 10-35\% improvement in the throughput of the decentralized network and 40-90\% reduction in the number of subband switchings compared to existing DMPs. The simulation results are then validated using real radio signals on the proposed USRP testbed.
\end{abstract}

Keywords: Decision making policy, multi-armed bandit algorithms, radio

\footnotetext{
*Corresponding author

Email addresses: Sumit@iitt.ac.in (Sumit J. Darak), honggangzhang@zju.edu.cn

(Honggang Zhang), Jacques.Palicot@centralesupelec.fr (Jacques Palicot),

Christophe.Moy@centralesupelec.fr (Christophe Moy)
} 
frequency energy harvesting, Thompson sampling.

\section{Introduction}

Opportunistic spectrum access (OSA) based cognitive radio (CR) paradigm has received significant attention from the academia as well as industrial partners [1-9]. In wireless networks, CR paradigm allows unlicensed users to com-

5 municate over identified vacant licensed subband(s). In some cases, unlicensed users do not need base stations or access points for establishing the communication link. This is termed as underlay inband communications [1,2]. OSA based Long Term Evolution-Unlicensed (LTE-U) has been demonstrated as potential and feasible way to improve the throughput of wireless networks. Furthermore, some data intensive and delay tolerant services in the LTE-Advanced and 5G may use OSA to meet their spectrum needs and improve the network throughput $[10,11]$. The popular schemes for such OSA communications are: 1) Centralized scheme where base station is responsible for orthogonalization of CR terminals to optimum vacant frequency bands, and 2) Decentralized scheme where CR 15 terminals independently choose the vacant subband(s) without an explicit information exchange or pre-agreement among them. Decentralized approach would be an efficient choice over the centralized approach for larger networks due to advantages such as ease of implementation, robustness to link or node failures, no communication overhead and lower delay $[1,2]$. Furthermore, decentralized

${ }_{20}$ approach is the preferred choice for public safety networks and proximity-aware social networking services.

Energy harvesting from solar, thermal, radio frequency (RF) sources for green self-sustainable communications has recently generated lots of interest due to rising energy demand and cost, awareness towards clean environment

25 and green taxes etc. Such harvesting approaches improve the battery life of radio terminals making them self-sustainable up to certain extent. The RF energy harvesting (RFEH) is a new trending technique which facilitates the conversion of received RF signals into electricity that can be stored and used later for data transmission [3-9, 12]. Today, researchers have claimed that the 30 RF energy of $1 \mu \mathrm{W}$ to as high as $3.5 \mathrm{~mW}$ can be harvested at $2.4 \mathrm{GHz}$ and 900 $\mathrm{MHz}$ frequencies depending on the distance from the source $[3,6-9]$. In near future, further improvements in the RFEH circuits and an international RFEH standard can be expected.

Consider the decentralized wireless network consisting of multiple RFEH 35 enabled CR terminals as shown in Fig. 1. Primary users (denoted as PUs) are the licensed users which communicate with other PUs over the frequency bands allocated by base station. In CR mode, RFEH enabled CRs communicate directly with other CRs over the identified vacant frequency bands. In RFEH mode, CRs harvest the RF energy from sources such as base stations (denoted 40 as BS), WiFi access points and TV towers. Various decision making policies (DMPs) to identify the optimum vacant bands for CRs have been proposed in the literature [13-20]. Nonetheless, the subband selection task has become 
more challenging in the decentralized networks consisting of RFEH enabled CRs. This is because, occupied subbands with higher RF energy and wider contiguous bandwidth are the desired subbands in the RFEH mode while the vacant contiguous subbands of any tunable bandwidth and lower RF energy are the desired subbands in the CR mode (i.e., OSA mode). Furthermore, tunable vacant bandwidth requirements of CRs must be taken into account to support wide variety of services ranging from data to multimedia in 5G and LTE-A.

50 From power consumption perspective, the subband switching cost (SSC), i.e. total penalty incurred in terms of delay, power, hardware reconfiguration and protocol overhead when CR switches from one frequency subband to another, should be as minimum as possible. To the best of our knowledge, existing DMPs $[1-9,12,14-21]$ are not mature enough to provide the comprehensive 55 solutions for the subband selection, subband access and mode selection tasks for RFEH enabled CRs in the decentralized network. The design of such DMP is an objective of the work presented in this paper.

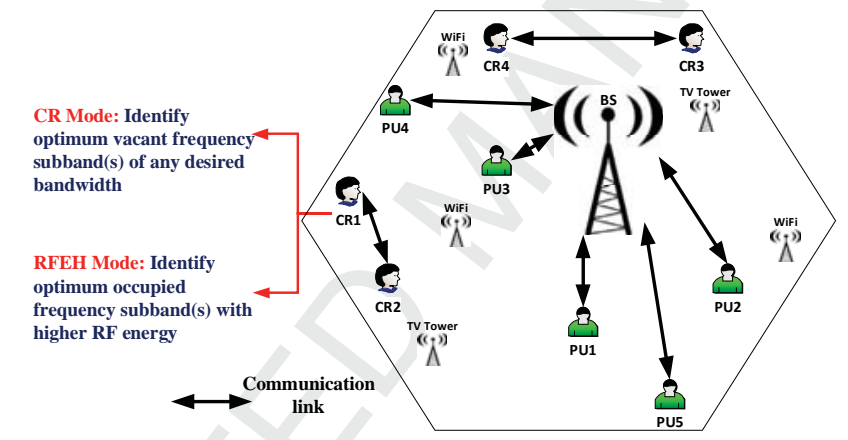

Figure 1: A decentralized wireless network with RFEH enabled CR terminals and RF energy sources.

In this paper, a new CR-RFEH DMP, a significant extension of our work in [13], has been proposed. The proposed DMP consists of: 1) Tunable Thomp60 son Sampling (TTS) algorithm to estimate the subband statistics for CR as well as RFEH modes, 2) Subband access scheme for orthogonalization of CRs in distinct subbands to minimize the number of collisions, and 3) Mode selection scheme to switch modes i.e., CR to RFEH and vice-versa. To the best of our knowledge, the proposed DMP is the first one designed using Bayesian ${ }_{55}$ approach based TS algorithm compared to existing DMPs designed using frequentist approach based learning algorithms for the subband statistic estimation $[16,17,19,20]$. Furthermore, the proposed DMP takes into account the specific subband requirements of the RFEH mode and tunable bandwidth access in the CR mode. Another novelty is that the proposed DMP uses TS algorithm for the

70 subband access scheme compared to the randomization approach based schemes in $[16,17,19,20]$. The detailed simulation as well as experimental results on the USRP testbed validate the superiority of the proposed DMP over existing DMPs. 
The paper is organized as follows. The detailed literature review is given in Section 2. The proposed DMP is presented in Section 3 followed by the simulation results in the Section 4. In Section 5, proposed USRP testbed is presented along with the experimental results and performance comparisons of various DMPs. Section 6 concludes the paper.

\section{Literature Review}

In this section, a review of existing DMPs for the decentralized wireless networks is presented.

\subsection{DMP for Decentralized Networks without RFEH}

In [14-20], decentralized wireless networks consisting of CR terminals without RFEH capability are considered. In such CRs, DMP operates only in one mode, i.e., CR mode. For networks where subband statistics are not known, they are estimated using learning algorithms such as frequentist approach based upper confidence bound (UCB) algorithm and its extensions, $\varepsilon$ - greedy, KullbackLeibler UCB (KL-UCB) etc. [14-20]. Such algorithms, also known as multiarmed bandit (MAB) algorithms, are based on exploration-exploitation dilemma

90 i.e., the trade-off between the exploration of all subbands to accurately estimate their statistics and selecting the subband with the desired statistic, i.e., the subband with the highest probability of being vacant. MAB algorithms are asymptotically optimal with logarithmic regret. Here, the regret means the loss of data transmission opportunities due to the selection of sub-optimal subbands. Re95 cently, Bayesian MAB algorithms such as TS and Bayes-UCB have been proved to have better regret than others and are discussed later in Section 3.2.

Even after the accurate estimation of subband statistics, DMPs in the decentralized network can incur significant regret due to the collision among CRs. To minimize the number of collisions, subband access scheme is desired for 100 orthogonalization of CRs. The design of the subband access scheme for the decentralized networks is non-trivial since CRs do not share information with each other. In $\rho^{\text {rand }}$ scheme for the decentralized network with $M$ CRs [16], each CR randomly and independently chooses the rank, $R(k) \in\{1,2, . . M\}$ in the beginning. In the subsequent time slots, the underlying MAB algorithm calculates the quality index, based on past sensing events, for each subband. Then, CR with the rank $R(k)$ selects the subband with the $R(k)^{t h}$ best quality index. Another policy in [17] follows time division fare share approach where the rank of each $\mathrm{CR}$ is rotated in circular fashion between 1 to $M$ to allow an equal access to the optimum subbands to all CRs. In [16, 17], a new rank is randomly and independently chosen for CR experiencing collision. Though both DMPs are optimal with logarithmic regret, SSC in [17] increases linearly with $t$. The SSC can be minimized using the policy, $\rho^{\text {rand }}[16]$, where the rank changes only when corresponding CR collides with other CRs. The $\rho^{\text {rand }}$ is further improved in $[18,19]$. In [18], the number of collisions are reduced by allowing the rank to be greater than $M$. In [19], access strategies in [16, 17] are combined with MAB 
algorithms to take into account different subband statistics. However, the SSC of [19] is high for the subband statistics where it performs superior than [16]. In [20], variable filtering architecture and its integration with the tunable subband access policy, $\rho^{\text {t_rand }}$, is presented that takes into account tunable bandwidth requirements of CRs. Still, the average SSC of [18-20] is high.

\subsection{DMP for Decentralized Networks with RFEH Capability}

Recent research efforts $[3-9,12]$ investigating the different ways to adopt $\mathrm{RFEH}$ in the wireless networks can be classified broadly into two categories. The first category is related to the design of RFEH circuits using single band, dual band or broadband antennas and their integration with CR terminals. Detailed discussion on these issues is out of the scope of this paper and reader can refer to $[3,6-9]$ for more information. To summarize, the amount of harvested RF energy increases with the increase in the strength of the RF signal, RFEH duration as well as harvesting bandwidth [3, 6-9]. Furthermore, among various harvesting approaches including solar, thermal etc., RFEH circuits have higher number of circuit elements with are present in the AFE of the existing CR terminals making RFEH a preferred choice from architecture perspective.

The second category deals with the design of DMPs for the decentralized network consisting of RFEH enabled CRs. Due to additional RFEH mode, DMPs for RFEH enabled CRs involve more complex decision making compared to DMPs discussed in Section 2.1. In [4], two schemes, namely time-splitting and power-splitting, have been proposed for information as well as RFEH cooperation between primary users and CRs. In the time-splitting scheme, CR and RFEH modes are separated in time while in the power-splitting approach, Rs can simultaneously extract RF energy and information from the received PU signals. In turn, CRs must help primary users for data relaying or offloading. Though power-splitting scheme is efficient in theory, it has drawbacks such as hardware complexity, incompatibility with existing circuits and unsuitable for out-of-band RFEH. The usefulness of these schemes for large decentralized networks and ambient RFEH is contentious.

The DMP in [5] deals with the switching actions between CR and RFEH modes and the goal is to maximize overall throughput of the decentralized network. However, subband statistics are known in advance which may not be true for the decentralized networks. In [12], a new frame structure for RFEH enabled CRs to perform harvesting, spectrum sensing and data transmission in single time slot is proposed. Such approach may not be feasible with existing RFEH circuit due to poor efficiency. The optimization based policy in [6] and learning based policy in [7] consider the subband selection for RFEH enabled CRs in the decentralized networks. Though both policies are proved to be optimal, they are designed for networks with single CR which means that the collisions among CRs, a significant factor affecting the subband selection and hence, total regret of the practical decentralized networks, has not been considered. An optimization based DMPs are computationally complex and may not be feasible for battery-operated resource-constrained CR terminals. Hence, a learning 
DMPs, it is assumed that $\theta \approx 1$ (Here, $\theta$ denotes the number of successful RFEH time slots needed for harvesting the RF energy sufficient for single data transmission over the same bandwidth). Considering a feasible range of $\theta$ from 4 to 15 for existing RFEH circuits [8,9], with the likelihood of smaller $\theta$ in near future, the effect of $\theta$ on the total regret of the decentralized networks has not been analyzed yet in the literature.

\section{Proposed CR-RFEH Decision Making Policy}

Consider a feasible architecture of RFEH enabled CR as shown in Fig. 2. It mainly consists of

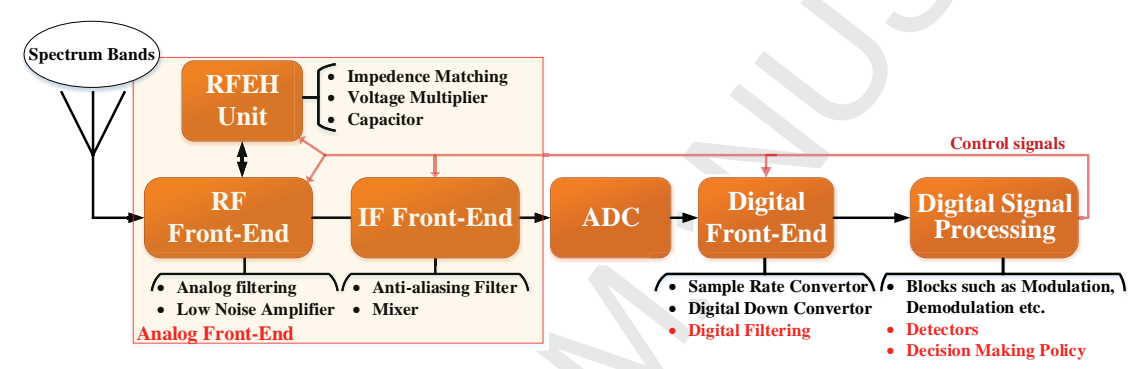

Figure 2: A feasible architecture of RFEH enabled CR terminal.

1. Analog and digital front-end: An analog front-end receives wideband input signal from antenna and performs the tasks such as RF to intermediate frequency (IF) conversion, filtering to prevent aliasing, signal amplification etc. ADC performs analog IF to digital IF conversion. The digital frontend, consisting of digital filtering and signal processing algorithms, performs various tasks as shown in Fig. 2.

The total SSC is the product of the number of subband switchings and the penalty due to single subband switching. The number of subband switchings depend on the DMP while single subband switching penalty can be further divided into two parts: 1) Penalty due to the hardware (mainly analog and digital filters) reconfiguration, and 2) Communication cost since the receiver needs to be informed about the change in subband. Taking into account inter-standard as well as intra-standard bandwidth variations and limited flexibility of the analog filters compared to its digital counterpart, the analog front-end performs only coarse filtering task and the digital front-end is responsible for the stringent subband filtering. Furthermore, in multi-standard environment, input signal comprises of multiple communication standards each with distinct bandwidth specifications. In such cases, the digital filtering stage needs variable digital filters which offer complete control over the bandwidth and center frequencies over the entire Nyquist band. Hence, a 
2. Each CR operates either in CR mode or RFEH mode in a given time slot. The mode selection scheme independently chooses the mode for each CR.

3. CR can sense only one subband in a time slot.

4. Subbands occupied by CRs are not useful for RFEH due to lower levels of RF energy.

5. Subband statistics are fixed, stationary and unknown to CRs. 
Consider $i^{\text {th }}$ subband switches its state from being vacant to occupied and vice-versa according to a discrete Markov process with the probabilities of $p_{v o}^{i}$ and $p_{o v}^{i}$, respectively. Then, using Markov chain analysis, steady-state probabilities of subband being vacant, denoted as $P_{v a c}(i)$, are given by $[5,7]$

$$
P_{v a c}(i)=\frac{p_{o v}^{i}}{p_{o v}^{i}+p_{v o}^{i}}, \forall i \in\{1,2, \ldots N\}
$$

In $\mathrm{CR}$ mode, chosen subband(s) are filtered by the digital filtering stage and checked for vacancy in the detection stage. If vacant, it is assumed that the CR transmits over that subband(s). When multiple CRs transmit on the subbands which are non-orthogonal in frequency, collision occurs leading to failed transmission. When no such collision occurs, it is assumed that the CR transmits successfully. Then, the reward of $k^{\text {th }} \mathrm{CR}$ at time $t, \nabla(k, t)$, depends on the amount of the data transmitted by $\mathrm{CR}$ and is given by

$$
\nabla(k, t)= \begin{cases}\frac{B_{v}(k)}{B_{c m i n}} & \text { No collission } \\ 0 & \text { Collision/RFEH mode }\end{cases}
$$

Let $S^{*}(t)$ and $S(t)$ denote the total reward (i.e., throughput) of the network using the genie-aided policy (i.e. the policy where $P_{v a c}(i), \forall i$, are known a priori and a central unit ensures orthogonal optimum subband allocation to CRs in the CR mode) and the decentralized DMP, respectively. Then, total regret, $U(t)$, up to time $t$ is given by Eq. $3[14-18,20]$ and it should be as minimum as possible.

$$
U(t)=S^{*}(t)-S(t)=\sum_{k=1}^{M} \sum_{v=0}^{t-1}\left[\nabla^{*}(k, v)-\nabla(k, v)\right]
$$

Let $\xi(k, t)$ be the available battery charge of $k^{t h} \mathrm{CR}$ at time $t$. If $\xi_{d}$ is the battery charge consumed by $\mathrm{CR}$ for each data transmission, then

$$
\xi(k, t)=\xi(k, t-1)-\xi_{d}
$$

In RFEH mode, RF energy of the chosen subband(s) is harvested by RFEH circuit. Let $\xi_{e}$ be the battery charge from the energy harvested by RFEH circuit over the chosen subband, then

$$
\begin{gathered}
\xi(k, t)=\xi(k, t-1)+\xi_{e} \\
\xi_{d}=\theta \cdot \xi_{e}
\end{gathered}
$$

where $\theta$ represents the number of time slots over which RFEH needs to be done in order to harvest energy required for single data transmission. Parameter, $\theta$, which depends on the available RF energy and efficiency of RFEH circuit, should be as minimum as possible. From the existing RFEH circuit specifications $[3,6-9]$, it can be safely assumed that $\theta \geq 4$. Note that harvesting and data transmission bandwidths are assumed to be equal in Eq. 6. In Table 1, all the notations used in this paper are given along with their definitions. 


\begin{tabular}{|c|c|}
\hline Notations & Definitions \\
\hline$M$ & No. of CRs in the decentralized network \\
\hline$N$ & No. of subbands of bandwidth $B_{c m i n}$ \\
\hline$N_{d}(k)$ & No. of subbands of bandwidth $B_{v}(k)$ \\
\hline$N_{r f}$ & No. of subbands of bandwidth $B_{a f e}$ \\
\hline$B_{c m i n}$ & Smallest subband bandwidth \\
\hline$B_{a f e}$ & Analog front-end bandwidth of CR terminal \\
\hline$B_{v}(k)$ & Desired bandwidth of $k^{t h} \mathrm{CR}$ in CR mode \\
\hline$B_{o}(k)$ & Desired bandwidth of $k^{\text {th }} \mathrm{CR}$ in RFEH mode \\
\hline$P_{v a c}(i)$ & Probability of vacancy of subband $i$ \\
\hline$R(k)$ & Rank of $k^{t h} \mathrm{CR}$ \\
\hline$I_{d}(k, t)$ & Subband chosen by $k^{\text {th }} \mathrm{CR}$ in the CR mode \\
\hline$I_{r f}(k, t)$ & Subband chosen by $k^{\text {th }} \mathrm{CR}$ in the RFEH mode \\
\hline$\nabla_{d}(k, t)$ & Reward of $k^{\text {th }} \mathrm{CR}$ in the CR mode \\
\hline$\nabla_{r f}(k, t)$ & Reward of $k^{t h}$ CR in the RFEH mode \\
\hline$\nabla_{r}(k, t)$ & Reward of $k^{t h}$ CR using the rank $R(k)$ \\
\hline$T_{d}(k, i, t)$ & No. of times the subband $i$ is chosen by $k^{t h} \mathrm{CR}$ in the $\mathrm{CR}$ mode \\
\hline$T_{r f}(k, i, t)$ & No. of times the subband $i$ is chosen by $k^{t h} \mathrm{CR}$ in the RFEH mode \\
\hline$T_{r}(k, i, t)$ & No. of times the rank $i$ is chosen by $k^{\text {th }} \mathrm{CR}$ in the $\mathrm{CR}$ mode \\
\hline$r_{d}(k, i, t)$ & Total reward of $k^{t h} \mathrm{CR}$ using the subband $i$ in the CR mode \\
\hline$r_{r f}(k, i, t)$ & Total reward of $k^{t h} \mathrm{CR}$ using the subband $i$ in the RFEH mode \\
\hline$r_{r}(k, i, t)$ & Total reward of $k^{t h} \mathrm{CR}$ using the rank $i$ in the CR mode \\
\hline$c_{r}(k, i, t)$ & No. of collisions experienced by $k^{\text {th }} \mathrm{CR}$ using the rank $i$ \\
\hline$X_{d}(k, i, t)$ & Estimated vacancy statistics of $i^{\text {th }}$ subband by $k^{\text {th }} \mathrm{CR}$ \\
\hline$X_{r f}(k, i, t)$ & Estimated RF potential of $i^{t h}$ subband by $k^{t h} \mathrm{CR}$ \\
\hline$S(t)$ & Total reward (i.e., throughput) of the DMP \\
\hline$S^{*}(t)$ & Total reward (i.e., throughput) of the genie aided DMP \\
\hline$U(t)$ & Total regret of the DMP w.r.t. the genie aided DMP \\
\hline$R_{m}(k)$ & Upper limit on the rank for $k^{\text {th }} \mathrm{CR}$ \\
\hline$\xi(k, t)$ & Available battery charge of $k^{t h} \mathrm{CR}$ \\
\hline$\xi_{p}(k, t)$ & Available battery charge of $k^{t h} \mathrm{CR}$ in $\%$ \\
\hline & Charge consumed by CR for single data transmission \\
\hline$\xi_{e}=\xi_{d} / \theta$ & Charge harvested by CR after successful energy harvesting \\
\hline$P_{d}$ & Probability of $\mathrm{CR}$ to remain in the $\mathrm{CR}$ mode \\
\hline$P_{r f}$ & Probability of CR to remain in the RFEH mode \\
\hline
\end{tabular}

\subsection{Proposed CR-RFEH DMP: Subband Statistic Estimation}

Various tasks of the proposed CR-RFEH DMP are shown in Fig. 3. Each time slot is divided into two sub-slots, $t_{s}$ and $t_{d}$. In slot $t_{s}$, mode selection, sub- 
band selection and subband statistics update take place while the data transmission (in CR mode) and energy harvesting (in RFEH mode) over the chosen subband is done in slot, $t_{d}$.

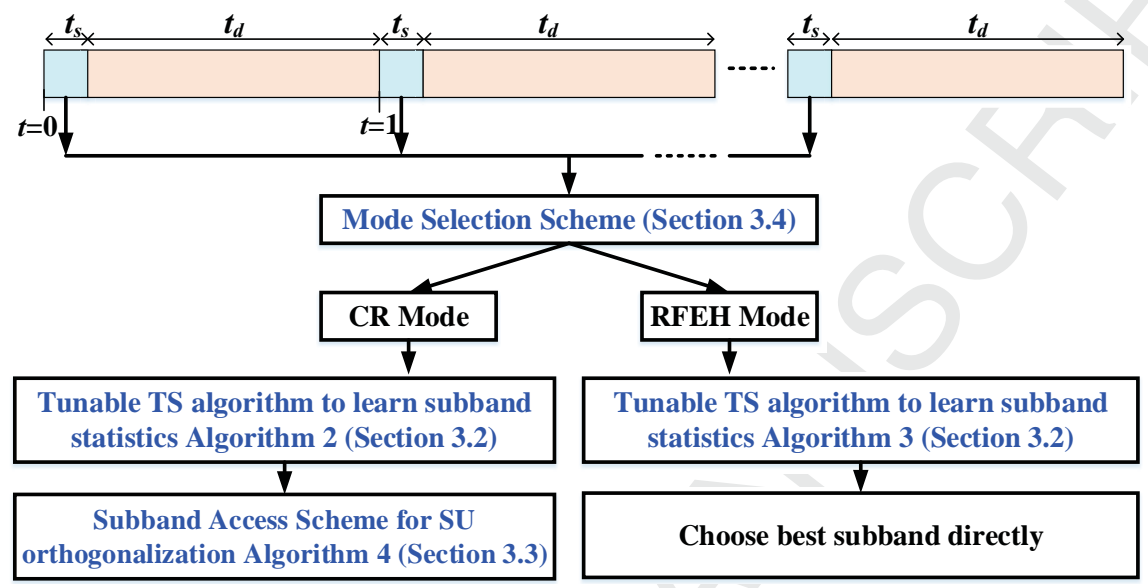

Figure 3: Different tasks of the proposed CR-RFEH policy

First contribution of this paper is the selection of Bayesian MAB algorithm for subband statistic estimation. To begin with, Bayesian MAB algorithm is discussed followed by its advantages over other algorithms. The basic idea of Bayesian MAB algorithms is to assume some prior distribution on the probability statistics of each subband (e.g. uniform prior) and at any time slot, select the subband according to its posterior probability of being optimum [27-29]. Among them, TS algorithm [27-29] has been chosen for the proposed CR-RFEH DMP and its usefulness for the subband statistic estimation is given in Algorithm 1. In the beginning, all subbands are chosen once as shown in steps 1-2. Then, TS algorithm calculates the quality index for each subband based on the past sensing events as shown in steps 5-6. Here, the horizon, H, represents the total number of time slots for which the DMP is executed and Beta represents the complete beta function, i.e., Euler integral of the first kind. Then, CR with the rank $R(k)$ selects the subband having $R(k)^{t h}$ highest quality index. In Algorithm 1 , parameter $T(k, i, t)$ denotes the number of time slots up to $t$ slots where the subband $i$ is sensed by $k^{t h} \mathrm{CR}$ and $r(k, i, t)$ denotes the number of times out of $T(k, i, t)$ slots, the chosen subband $i$ is observed as vacant. The advantages of TS algorithm over other MAB algorithms can be summarized as follows:

1. MAB algorithm is said to be optimal in the subband selection task if [27-29]

$$
\liminf _{t \rightarrow \infty} \frac{\mathbb{E}[T(k, i, t)]}{\ln t} \geq \frac{1}{K\left(P_{\text {vac }}(i), P_{\text {vac }}\left(i^{*}\right)\right)}, \forall i
$$




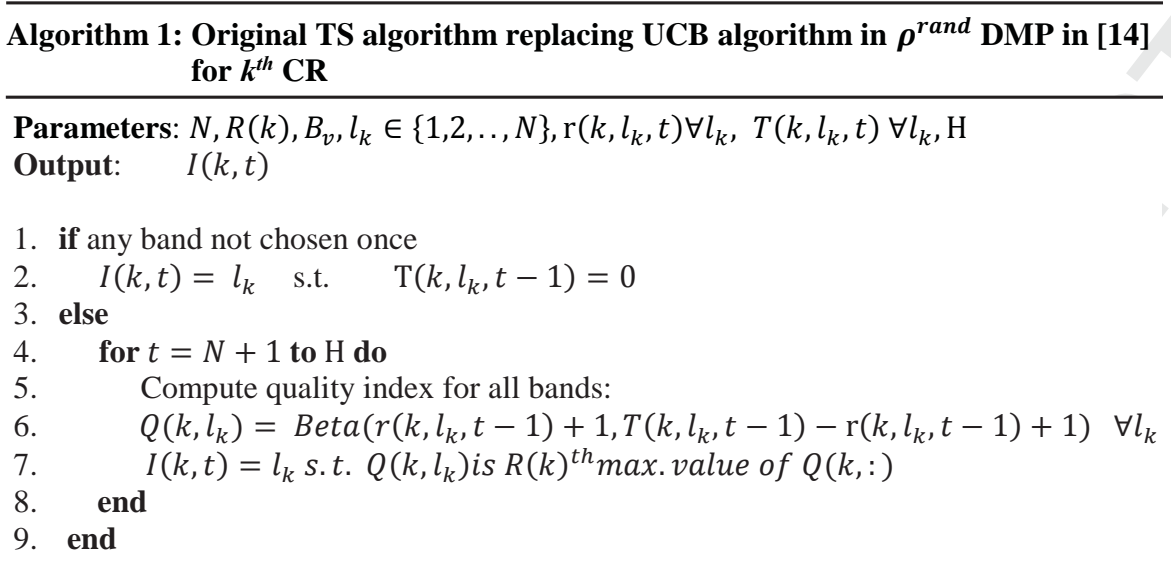

where

$$
i^{*}=\arg \max P_{v a c}(i)
$$

where $K(p, q)$ denotes the Kullback-Leibler divergence factor. The algorithm which satisfies Eq. 7 with equality is said to be asymptotically optimal. Frequentist approach based UCB algorithms, optimization based KL-UCB algorithm [29], Bayesian approach based TS and Bayes-UCB algorithms [27] have been showed to be asymptotically optimal for Bernoulli rewards. However, it has been recently proved that the scaling constant and hence, total reward of the Bayesian algorithms is better than that of others [27, 28].

2. TS algorithm requires the computation of only one sample from the posterior of subband statistics as shown in Algorithm 1. Its complexity is low compared to optimization problem in KL-UCB algorithm and computation of quantiles in Bayes-UCB algorithm [27-29].

3. Bayesian subband selection approach leads to lower SSC than UCB algorithm or its extensions through empirical observations.

To the best of our knowledge, usefulness of TS algorithm, rather any Bayesian $\mathrm{MAB}$ algorithm, for the decentralized networks or any other wireless communication applications has not been studied yet. Furthermore, existing TS algorithm needs to be re-designed to take into account tunable bandwidth scenarios, i.e., $B_{v}(k)>B_{c m i n}$. For example, in $\mathrm{CR}$ mode, the number of subband choices available to CRs, $N_{d}(k)$, are given by

$$
N_{d}(k)=N+1-\frac{B_{v}(k)}{B_{c m i n}}
$$

When $B_{v}(k)=B_{c m i n}, \forall k$, all the subbands are orthogonal in frequency. Thus, CRs with distinct ranks do not experience any collision [16-19]. How- 
ever, when $B_{v}(k)>B_{c m i n}, \forall k$, each subband is orthogonal to all other subbands except adjacent $\left(\frac{B_{v}(k)}{B_{c m i n}}-1\right)$ number of subbands. Thus, CRs with distinct ranks may also experience collision due to non-orthogonality of subbands. For example, when $N=4, M=2$ and $B_{v}=\{2,2\}$, then $N_{d}(k)=3 \forall k$. This means that CRs have three subband choices which are contiguous subbands $\{1,2\},\{2,3\}$ and $\{3,4\}$. For example, $k^{\text {th }} \mathrm{CR}$ with rank $R(k)=1$ will choose contiguous subband $\{1,2\}$. Thus, even though two CRs have distinct ranks, say 1 and 2 , they will experience collision due to common subband 2. Furthermore, the probability of collision increases with the bandwidth, $B_{v}(k)$ compared to [16-19] where $B_{v}(k)=B_{c m i n}, \forall k$ and hence, probability of collision is fixed for all CRs. By taking these factors into account, tunable TS (TTS) algorithm is proposed for CRs in the CR mode and is given below in Algorithm 2.

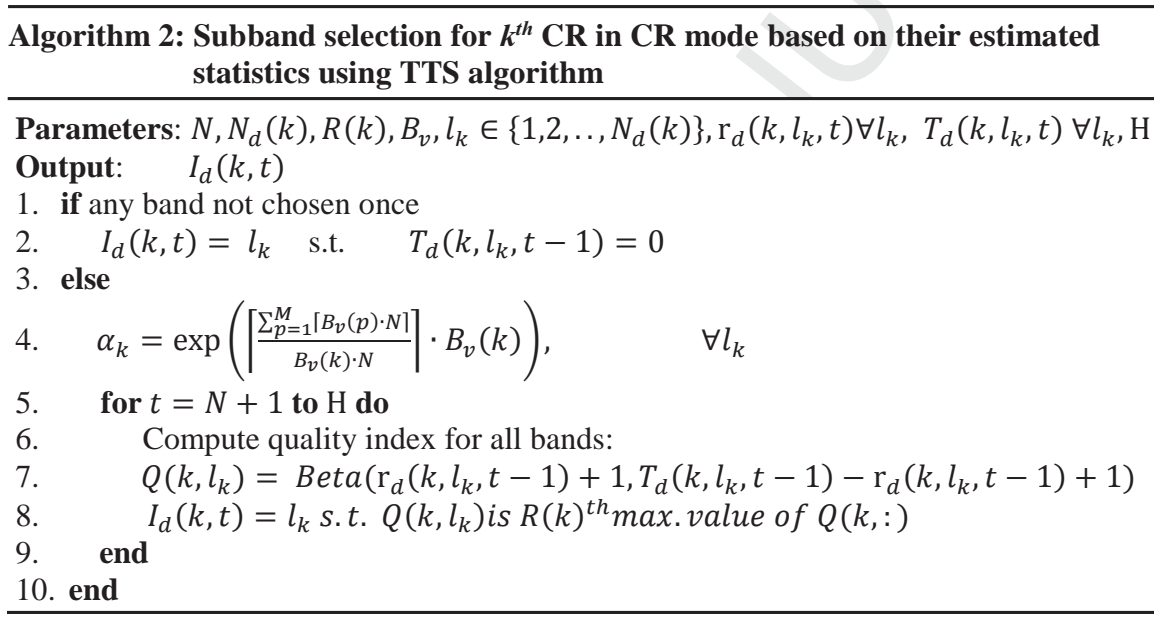

In TTS algorithm, the parameter, $\alpha_{k}$, is introduced to minimize the number of collisions due to non-orthogonality of subbands and the rank, $R(k)$ is obtained using the scheme discussed later in Section 3.3. If the chosen subband, $I_{d}(k, t)$ is sensed as vacant by $k^{t h} \mathrm{CR}$ at time $t$, then $\nabla_{d}(k, t)=1$, otherwise $\nabla_{d}(k, t)=0$. Based on $\nabla_{d}(k, t), r_{d}\left(k, l_{k}, t\right)$ is updated as

$$
\begin{gathered}
r_{d}\left(k, I_{d}(k, t), t\right)=r_{d}\left(k, I_{d}(k, t), t-1\right)+\nabla_{d}(k, t) \\
r_{d}\left(k, l_{k}, t\right)=r_{d}\left(k, l_{k}, t-1\right) \quad \forall l_{k} \neq I_{d}(k, t)
\end{gathered}
$$

On the other hand, if the chosen subband, $I_{d}(k, t)$, is observed as occupied with RF energy higher than the threshold, $\Gamma_{r f}$, then $\nabla_{r f}(k, t)=1$, otherwise $\nabla_{r f}(k, t)=0$. Note that the threshold, $\Gamma_{r f}$, depends on the static power consumption of RFEH circuit. Then, $r_{r f}\left(k, l_{k}, t\right)$, to be used later in the RFEH mode and analogous to $r_{d}\left(k, l_{k}, t\right)$ in the CR mode, is updated as

$$
r_{r f}\left(k, I_{d}(k, t), t\right)=r_{r f}\left(k, I_{d}(k, t), t-1\right)+\nabla_{r f}(k, t)
$$




$$
r_{r f}\left(k, l_{k}, t\right)=r_{r f}\left(k, l_{k}, t-1\right) \quad \forall l_{k} \neq I_{d}(k, t)
$$

In this way, the proposed DMP uses single TTS algorithm to learn the subband statistics for the CR as well as RFEH modes. Let $X_{d}\left(k, l_{k}, t\right)$ denotes the learned probability of $l_{k}^{t h}$ subband being vacant at $k^{t h}$ CR. Similarly, $X_{r f}\left(k, l_{k}, t\right)$ denotes the learned probability of $l_{k}^{t h}$ subband having RF energy higher than $\Gamma_{r f}$ at $k^{t h} \mathrm{CR}$. Then,

$$
\begin{gathered}
X_{d}\left(k, l_{k}, t\right)=\frac{r_{d}\left(k, l_{k}, t\right)}{T_{d}\left(k, l_{k}, t\right)}, \forall l_{k} \\
X_{r f}\left(k, l_{k}, t\right)=\frac{r_{r f}\left(k, l_{k}, t\right)}{T_{d}\left(k, l_{k}, t\right)}, \forall l_{k}
\end{gathered}
$$

where

$$
T_{d}\left(k, l_{k}, t\right)=\sum_{p=1}^{t} 1_{\left\{I_{d}(k, p)=l_{k}\right\}}
$$

In the RFEH mode, $R(k)=1, \forall k$ since there are no collisions among CRs and the task of the TTS algorithm is to identify contiguous set of occupied subbands with higher RF energy. Contiguous bandwidth, $B_{o}(k)$, in the RFEH mode is equivalent to $B_{v}(k)$ in the $\mathrm{CR}$ mode but $B_{v}(k)$ is chosen by CR depending on their transmission requirements while $B_{o}(k)$ is fixed and equal to the analog front-end bandwidth, $B_{a f e}$. This is because, the wider the bandwidth, the higher is the harvested RF energy [3, 6-9]. Then, the number of choices, $N_{r f}$, available for the $\mathrm{CR}$ in the RFEH mode are given by,

$$
N_{r f}=N+1-\left(\frac{B_{a f e}}{B_{c m i n}}\right)
$$

310 where $N_{r f}=N$ when $B_{a f e}=B_{c m i n}$ and $N_{r f}<N$ when $B_{a f e}>B_{c m i n}$. Note that $B_{a f e}$ is an integer multiple of $B_{c m i n}$. Let $m \in\left\{1,2, . ., N_{r f}\right\}$. Then, $m^{\text {th }}$ choice consists of contiguous subbands from $F(m)=m \in\{1,2, \ldots, N\}$ to $L(m)=m+B_{a f e}-1 \in\{1,2, \ldots, N\}$. For example, when $N=4$ and $B_{a f e}=$ $2 \cdot B_{\text {cmin }}$, then $N_{R F}=3, F=\{1,2,3\}$ and $L=\{2,3,4\}$. Then, the task of the 315 TTS Algorithm in the RFEH mode, given in Algorithm 3, is to identify the optimum subband (i.e., the subband with lower probability of being vacant and higher RF energy which will lead to faster RFEH). Similar to the CR mode, $r_{d}, r_{r f}, T_{r f}, X_{d}$ and $X_{r f}$ are updated in each time slot based on the sensing outcome of $I_{r f}(k, t)$. In this way, the TTS algorithm learns from the sensing outcomes in each mode.

In this paper, we have assumed that the subband statistics are fixed, stationary and unknown to CRs. This is an underlying assumption to estimate the subband statistics, $P_{v a c}$, based on the past sensing events. Similar assumption has also been made in the existing learning based DMPs [6, 7, 16-20, 27-29]. The proposed DMP can be extended to the slowly changing subband statistics, 
i.e., $P_{v a c}$ changes by small value after some finite but variable length time window. In such case, the TS algorithm needs to be modified to track the changes in the subband statistics. One approach is to calculate the quality index using the rewards received during the finite time window instead of infinite time window considered in the Algorithm 1 (and hence Algorithms 2 and 3). The length of the time window can be tuned based on the dynamic behavior of the subband statistics. Next, the subband access scheme of the proposed DMP is presented.

\subsection{Proposed CR-RFEH DMP: Subband Access Scheme}

The task of the subband access scheme, when implemented at all CRs in the decentralized network, is to orthogonalize CRs operating in the CR mode. Since there are no collisions among CRs in the RFEH mode, subband access scheme is not needed when the CR is in the RFEH mode. The proposed subband access scheme is based on the extension of our past works [13, 20] and involves two sub-tasks: 1) Calculation of the rank, $R(k)$, for each CR, and 2) Calculation of the upper limit of the rank, $R_{m}(k)$. The proposed solutions are discussed in the following sub-sections.

\subsubsection{MAB Algorithm Based Rank Calculation}

It is well known that the learning based subband selection schemes are superior to the random subband selection schemes [14-20]. Albeit, randomization based rank assignment approach has been used in the literature [16-20]. For example, in $[16,17,19]$, when collision occurs, corresponding CR randomly selects a new rank. Due to the random selection approach, there is a high probability of CR colliding with other CRs in the next time slot especially when $M$ is large. Thus, a single collision event leads to multiple collisions even after convergence especially when estimated subband statistics are not accurate. To alleviate this problem, a new rank assignment scheme using TS algorithm, replacing the con-

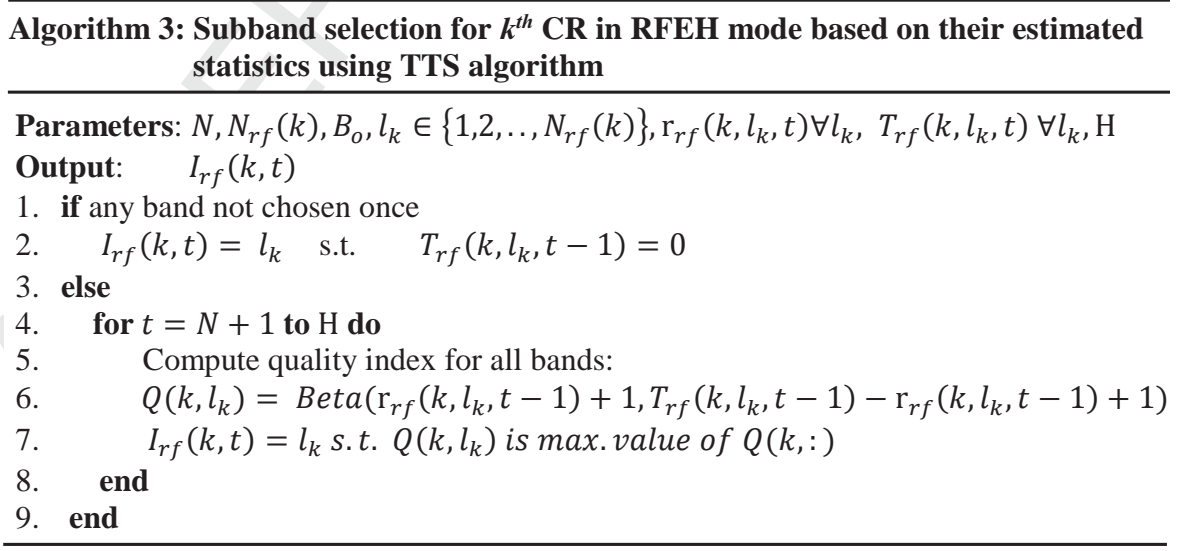


ventional random rank assignment scheme [16-20], is proposed and given in Algorithm 4.

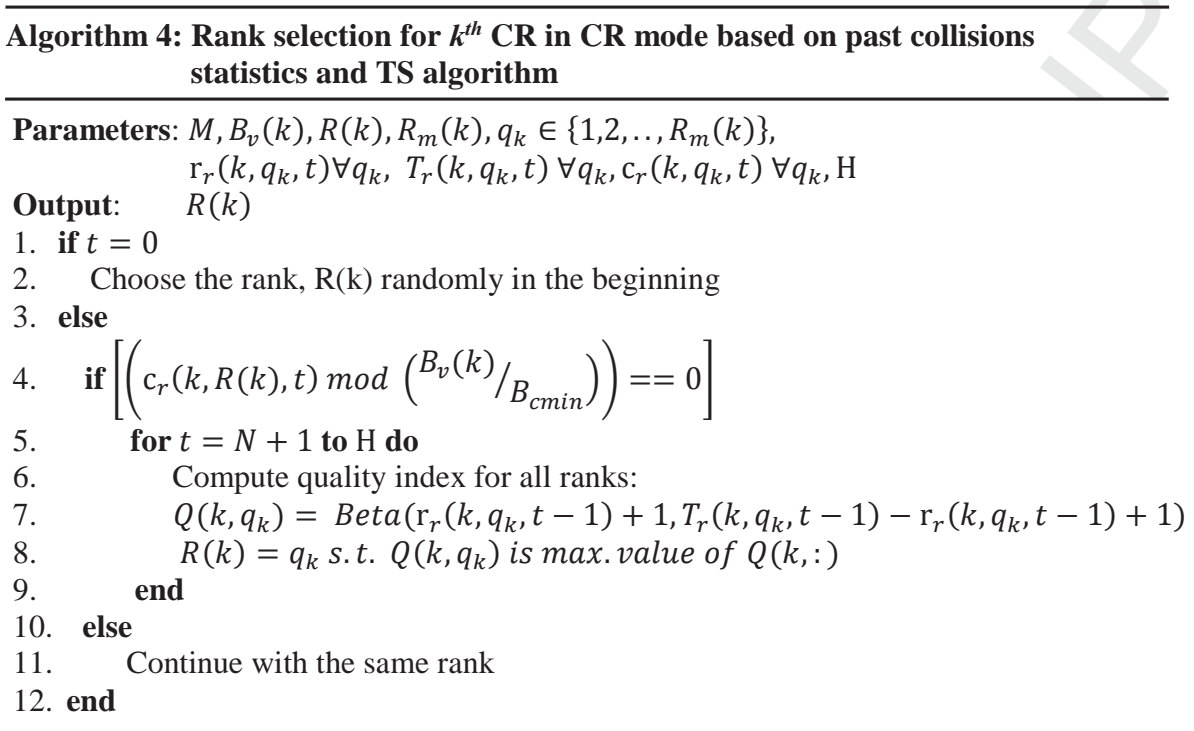

The task of the Algorithm 4 is to choose the rank, $R(k)$, for each CR in the CR mode based on the past collision events. This is analogous to the task of identifying optimum subband based on the past sensing events. In Algorithm 4, $T_{r}\left(k, q_{k}, t\right)$ denotes the number of time slots out of $t$ where $R(k)=q_{k}, q_{k} \in\left\{1,2, \ldots, R_{m}(k)\right\}$ and $c_{r}\left(k, q_{k}, t\right)$ denotes the number of time slots out of $T_{r}\left(k, q_{k}, t\right)$ where $k^{t h} \mathrm{CR}$ experiences collision on the rank, $q_{k}$. If the data transmission by $k^{\text {th }} \mathrm{CR}$ at time $t$ using the rank, $R(k)$ is successful, then $\nabla_{r}(k, t)=1$. Otherwise, $\nabla_{r}(k, t)=0$. Note that $\nabla_{r}(k, t)=1$ also means that the CR does not experience any collision. Then,

$$
\begin{gathered}
r_{r}(k, R(k), t)=r_{r}(k, R(k), t-1)+\nabla_{r}(k, t) \\
r_{r}\left(k, q_{k}, t\right)=r_{r}\left(k, q_{k}, t-1\right) \quad q_{k} \neq R(k) \\
c_{r}(k, R(k), t)=c_{r}(k, R(k), t-1)+1-\nabla_{r}(k, t) \\
c_{r}\left(k, q_{k}, t\right)=c_{r}\left(k, q_{k}, t-1\right) \quad q_{k} \neq R(k)
\end{gathered}
$$

In the proposed approach, the use of TS algorithm guarantees orthogonalization of CR in fewer time slots than $[16,17,19]$. Furthermore, the proposed approach also leads to fewer number of subband switchings (and hence, lower 
SSC) than $[16,17,19]$. The gain is significant for the tunable bandwidth access considered in this paper. This is because, the randomization based approach in $[16,17,19]$ leads to higher SSC when $B_{v}(k)>B_{\text {cmin }}$ as such CRs have higher probability of experiencing collisions. In the proposed scheme, first if loop in the Algorithm 4 eliminates this unfairness since the rank is updated only when the number of collisions past the recent rank assignment becomes

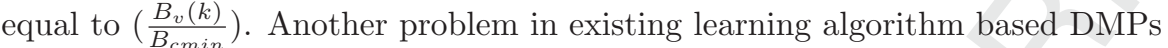
$[16,17,19]$ is that the number of collisions increases significantly as the number of CRs, $M$ increases. To alleviate this problem, the upper limit on the rank is made tunable as discussed below.

\subsubsection{Upper Limit on Rank, $R_{m}$}

In [20], we showed that the tunable upper limit of the rank leads to significant improvement in the performance of the randomization based subband access scheme when compared to the fixed subset size based schemes in $[16,17,19]$.

Taking it further, tunable upper limit on the rank, $R_{m}(k), \forall k$ for the proposed TS algorithm based subband access scheme is presented here. The lower and upper bounds on $R_{m}(k)$, denoted by $R_{m l}(k)$ and $R_{m u}(k)$ respectively, for $k^{t h}$ $\mathrm{CR}$, are given by,

$$
\begin{gathered}
R_{m l}(k)=\left\lceil\frac{\sum_{u=1}^{M}\left(\frac{B_{v}(u)}{B_{c m i n}}\right)}{\left(\frac{B_{v}(k)}{B_{c m i n}}\right)}\right\rceil, \forall k \\
R_{m u}(k)=N_{d}(k)=N+1-\frac{B_{v}(k)}{B_{c m i n}}, \forall k
\end{gathered}
$$

For example, when $N=6, M=2, B_{v}(1)=B_{c m i n}$ and $B_{v}(2)=2 \cdot B_{c m i n}$, first CR has six subband options, each of bandwidth $B_{c m i n}$ while second CR has five options, each of bandwidth $2 \cdot B_{c m i n}$. Then, $R_{m l}=\{3,2\}$ and $R_{m u}=\{6,5\}$. Note that $R_{m l}(k)=M$ for $B_{v}(k)=B_{c m i n} \forall k$, similar to the uniform bandwidth access considered in $[16,17,19]$. The optimum value of $R_{m}$, where $R_{m u} \geq R_{m} \geq$ $R_{m l}$, depends on the lower and upper bounds on the regret, $U(t)$. Such bounds for the tunable bandwidth case, using the bounds for uniform bandwidth case in $[16,17]$, are given as

$$
\begin{gathered}
U(t) \geq \sum_{k=1}^{M} \sum_{v=R_{m l}(k)+1}^{R_{m u}(k)} \Delta\left(k^{*}, v\right) \cdot \mathbb{E}(T(k, v, t)) \\
U(t) \leq \sum_{k=1}^{M} P_{v a c}\left(k^{*}\right)\left\{\sum_{v=R_{m l}(k)+1}^{R_{m u}(k)} \mathbb{E}[T(k, v, t)]+\sum_{u=1}^{R_{m l}(k)} \mathbb{E}\left[c_{d}(k, u, t)\right]\right\}
\end{gathered}
$$

where $c_{d}(k, u, t)$ denotes the number of time slots up to $t$ slots where $k^{\text {th }} \mathrm{CR}$ experiences collision while transmitting on the $u^{t h}$ subband of bandwidth $B_{v}(k)$, 
$P_{v a c}\left(k^{*}\right)$ denotes $k^{t h}$ highest value of $P_{v a c}$ and $\Delta\left(k^{*}, v\right)=P_{v a c}\left(k^{*}\right)-P_{v a c}(v)$

where $k^{*}$ is the subband of bandwidth, $B_{v}(k)$, with $k^{\text {th }}$ highest value of $P_{v a c}$. The upper bound on $U(t)$, given by Eq. 25, is of significant importance in order to reduce the number of collisions among CRs and hence, SSC. Eq. 25 consists of two terms: 1) First term represents the regret incurred due to selection of sub-optimum subbands, and 2) Second term represents the regret incurred due to collisions when optimum subbands are selected. For example, when $N=4, M=2, B_{v}=\left\{B_{c m i n}, B_{c m i n}\right\}$ and assuming that $P_{v a c}(1)>P_{v a c}(2)>$ $P_{v a c}(3)>P_{v a c}(4)$, the first term in Eq. 25 represents the regret due to selection of third and forth subbands and the second term in Eq. 25 represents the regret due to collisions when CRs select first and second subbands.

If $R_{m}(k) \approx R_{m l}(k)$ as in $[16,17]$, then the second term is dominant leading to the higher number of collisions among CRs and hence, higher regret, $U(t)$, as well as SSC. On the other hand, if $R_{m}(k) \approx R_{m u}(k)$, the first term is dominant leading to fewer number of collisions as well as lower SSC but it also leads to the higher regret, $U(t)$. Since, the loss in $U(t)$ due to the second term is more significant than the same due to the first term in the CR mode, a new method to make $R_{m}(k) \forall k$ tunable is proposed as follows:

1. For each integer value from $R_{m l}(k)$ to $R_{m u}(k)$, total predicted reward, $r_{t}\left(k, d_{k}, t\right) \forall k$ and $d_{k} \in\left\{1,2, . .,\left(R_{m u}(k)-R_{m l}(k)+1\right)\right\}$, at any time $t$ is given by,

$$
r_{t}\left(k, d_{k}, t\right)=\sum_{u=1}^{R_{m l}(k)+d_{k}-1} \frac{\bar{X}_{d}(k, u, t) \cdot R_{m l}(k)}{R_{m l}(k)+d_{k}-1}
$$

where $\bar{X}_{d}(k, u, t) \forall u$ is the estimated subband availability statistics, $X_{d}(k, u, t) \forall u$ in Eq. 14, arranged in the descending order, i.e., $\bar{X}_{d}(k, 1, t) \geq \bar{X}_{d}(k, 2, t) \geq$ $\cdots \geq \bar{X}_{d}\left(k, R_{m u}(k), t\right)$. It can be observed from Eq. 26 that $r_{t}\left(k, d_{k}, t\right)$ decreases as $d_{k}$ increases due to the selection of non-optimum subbands.

2. For each integer value from $R_{m l}(k)$ to $R_{m u}(k)$, total predicted number of collisions, $c_{t}\left(k, d_{k}, t\right) \forall k$ and $\forall d_{k}$, at any time $t$ is given by

$$
c_{t}\left(k, d_{k}, t\right)=1-\left[\frac{\left(R_{m l}(k)+d_{k}-1\right) !}{\left(d_{k}-1\right) ! \cdot\left(R_{m l}(k)+d_{k}-1\right)^{R_{m l}(k)}}\right]
$$

Eq. 27 indicates that the number of collision decreases as $d_{k}$ increases.

3. Finally, $R_{m}(k)$ is selected using the value of $d_{k}$ that leads to lower regret, $U(t)$ and is given by

$$
R_{m}(k)=R_{m l}(k)+s-1
$$

where

$$
s=\underset{d_{k}}{\arg \max }\left[r_{t}\left(k, d_{k}, t\right) \cdot t-c_{t}\left(k, d_{k}, t\right) \cdot t\right]
$$


Each CR independently calculates the value of $R_{m}(k)$. To minimize computational complexity, the value of $R_{m}(k)$ is updated only when corresponding CR updates its rank as discussed in Section 3.3.1. Next, the mode selection scheme is presented.

\subsection{Proposed CR-RFEH DMP: Mode Selection Scheme}

In the CR-RFEH DMP, each CR independently takes the mode selection decisions based on the available battery charge, $\xi_{p}(k, t)\left(0 \leq \xi_{p}(k, t) \leq 100, \forall k\right)$. Consider $P_{d}\left(\xi_{p}(k, t)\right)$ (or $P_{r f}\left(\xi_{p}(k, t)\right)$ denote the probability of $k^{t h}$ CR to remain in the CR mode (or RFEH mode, respectively) given that it is currently in the CR mode (or RFEH mode, respectively) and battery is $\xi_{p}(k, t) \%$ charged. Then,

$$
\begin{gathered}
P_{d}\left(\xi_{p}(k, t)\right)=1-P\left(\xi_{p}(k, t), \epsilon_{1}, \eta_{1}\right) \\
P_{r f}\left(\xi_{p}(k, t)\right)=P\left(\xi_{p}(k, t), \epsilon_{2}, \eta_{2}\right) \\
P\left(\xi_{p}(k, t), \epsilon, \eta\right)=\frac{\mathbb{S}\left(\xi_{p}(k, t)-\epsilon\right) \cdot\left|\xi_{p}(k, t)-\epsilon\right|^{\frac{1}{\eta}}+\left|\epsilon_{1}\right|^{\frac{1}{\eta}}}{\mathbb{S}(1-\epsilon) \cdot|1-\epsilon|^{\frac{1}{\eta}}+|\epsilon|^{\frac{1}{\eta}}}
\end{gathered}
$$

where $\mathbb{S}$ denotes the signum function, $\epsilon$ is the $\xi_{p}(k, t)$ at which there is a step change in $P$ and $\eta$ decides the slope of this step.

The illustrative plots of $P_{d}$ and $P_{r f}$ for the different combinations of $\left\{\epsilon_{1}, \epsilon_{2}, \eta_{1}, \eta_{2}\right\}$ are shown in Fig. 4. $P_{d}$ decreases while $P_{r f}$ increases when CR is in the CR mode. A step change in the values of $P_{d}$ and $P_{r f}$ can be seen at $\epsilon_{1}$ and $\epsilon_{2}$, respectively. Here, $\eta_{1}$ and $\eta_{2}$ control the values of $P_{d}$ and $P_{r f}$, respectively, at battery charges in the neighborhood of $\epsilon_{1}$ and $\epsilon_{2}$, respectively.

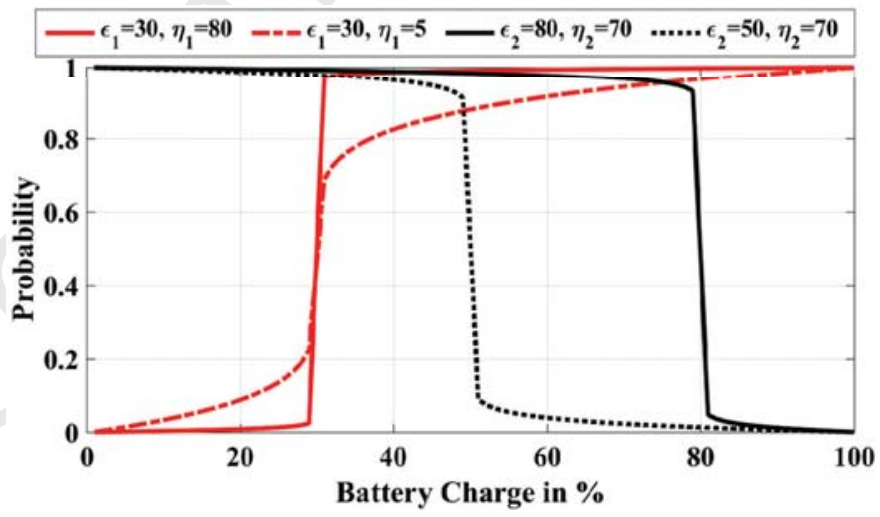

Figure 4: $\left\{P_{d}, P_{r f}\right\}$ vs. battery charge for different combinations of $\left\{\epsilon_{1}, \epsilon_{2}, \eta_{1}, \eta_{2}\right\}$.

Ideally, the total reward (i.e. throughput) of the DMP should be independent of $\left\{\epsilon_{1}, \epsilon_{2}\right\}$ values i.e. the mode switching frequency since many on-field factors such as location of $\mathrm{CR}$, priority of data transmission, available battery charge, 
type of service, future services and location etc. might influence the mode switching decision. Intuitively, this is true for the proposed CR-RFEH DMP since subbands statistics are assumed as stationary and the subband selection in each mode is based on the past sensing and collision events which means the mode switching events do not affect the performance of the DMP. This is verified using the simulation results presented in the Section 4 where the effects of $\left\{\epsilon_{1}, \epsilon_{2}, \theta\right\}$ on the reward and SSC of different DMPs are analyzed.

\section{Simulation Results}

In this section, we conduct extensive simulations to evaluate and compare the total reward and SSC of the proposed DMP with the $\mathrm{UCB}+\rho^{\text {rand }} \mathrm{DMP}$ in [16] and three other DMPs designed by replacing the UCB algorithm in [16] with TS, KL-UCB and Bayes-UCB algorithms, respectively. The mode selection scheme is same as Section 3.4 for all DMPs. Consider $N=16$ (i.e., $B_{\text {cmin }}=1 / 16$ ), $M \in\{3,4, . .12\}$ and three different distributions of $P_{v a c}$ which are

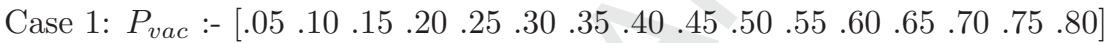

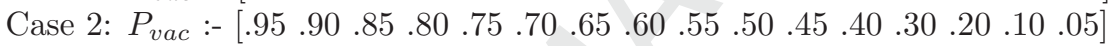

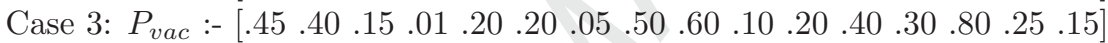

It is assumed that all CRs employ the same DMP but do not exchange any information with others. Each numerical result reported hereafter is the average of the values obtained over 50 independent experiments and simulations consider a time horizon of 50000 iterations.

To begin with, conventional CR based OSA in the decentralized network is considered. In such scenario, CR has unlimited battery capacity which means that the RFEH is not needed and CR has only one mode of operation i.e., CR mode. For $M=5$ and $B_{v}=\{1 / 16,3 / 16,2 / 16,2 / 16,1 / 16\}$, the plots of average reward, $S(t)$, in percentage vs. time slots, $t$, for Case 1 , 470 Case 2 and Case 3, are shown in Figures 5a, 5b and 5c, respectively. Here, $S(t) \%=\left[100 \cdot\left(S^{*}(t)-S(t)\right) / S^{*}(t)\right]$ where $S^{*}(t)$ is the average reward of the genie-aided policy. It is apparent from Fig. 5 that the proposed DMP outperforms other DMPs in terms of average reward for all $P_{v a c}$ distributions. Average reward is an indication of collision free data transmission opportunities offered by corresponding DMPs and hence, higher the reward, high is the throughput of the decentralized network. Fig. 5 shows that the average reward of the proposed DMP is significantly better than that of $\mathrm{TS}+\rho^{\text {rand }} \mathrm{DMP}$ which validates the superiority of the proposed TTS algorithm. Also, the proposed DMP outperforms other DMPs for each of the $P_{v a c}$ distributions which validates the superiority of the proposed TS algorithm based subband access scheme over the randomization based schemes [16-20].

Next, RFEH enabled CRs in the decentralized networks are considered. RFEH circuit parameters are $\theta=8$ (i.e., CR needs to perform RFEH for eight time slots over the bandwidth $B_{c m i n}$ in order to harvest energy required for single data transmission over the same bandwidth $B_{\text {cmin }}$ ) and $B_{\text {afe }}=3 \cdot B_{\text {cmin }}$. 


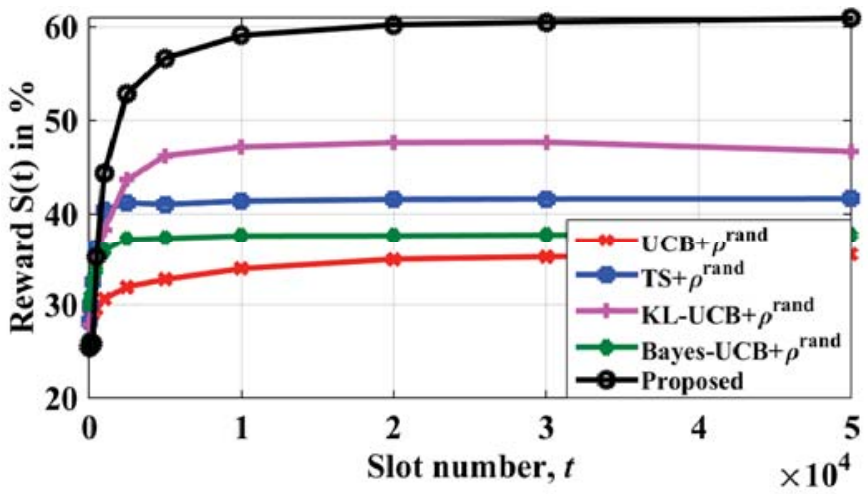

(a)

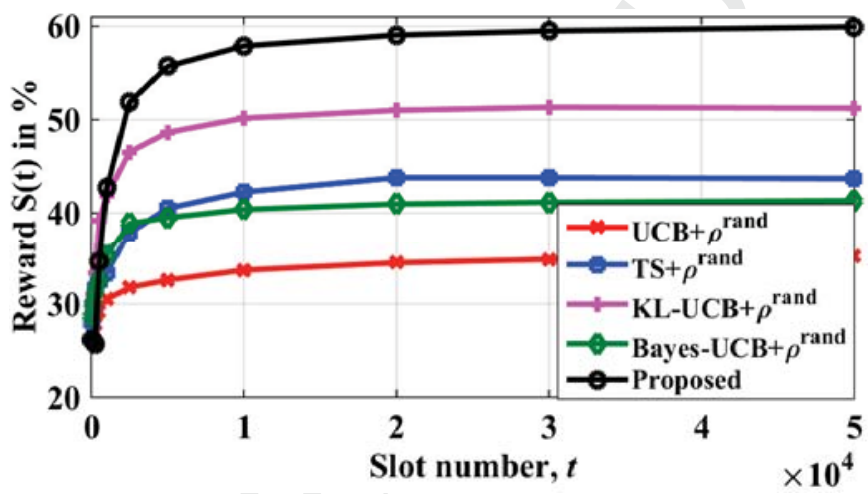

(b)

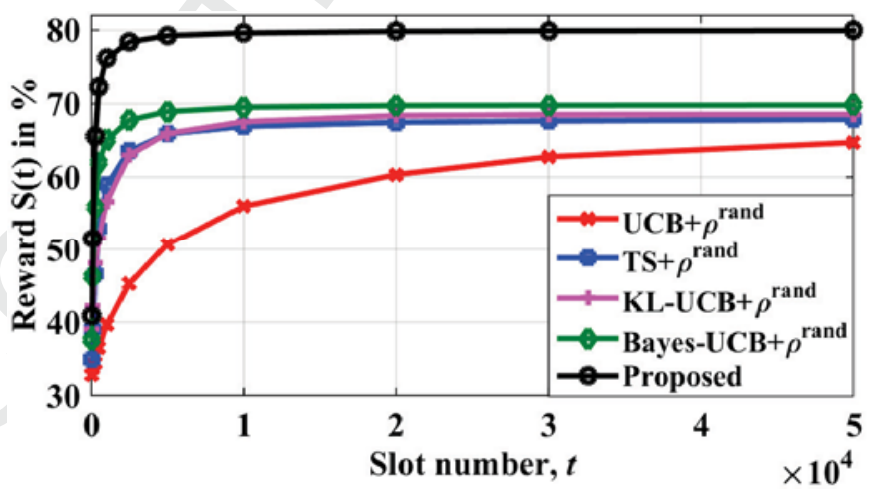

(c)

Figure 5: Average reward, $S(t)$ in $\%$ vs. time slot, $t$, for different $P_{v a c}$ distributions i.e., (a) Case 1, (b) Case 2, and (c) Case 3 in the conventional CR mode with no need of RFEH (only CR mode). 
Efficiency of RFEH circuits (i.e. the ratio of output DC power to the input RF power) varies non-linearly with input RF power and are taken from Powercast RFEH circuit specifications [30]. The parameters of the mode selection scheme, discussed in Section 3.4, are $\left\{\epsilon_{1}, \epsilon_{2}\right\}=\{20,30\}$ and $\left\{\eta_{1}, \eta_{2}\right\}=\{10,80\}$. It is assumed that the battery of each CR is $50 \%$ charged in the beginning. For $M=5$ and $B_{v}=\{1 / 16,3 / 16,2 / 16,2 / 16,1 / 16\}$, the plots of average reward, $S(t)$, in percentage vs. time slots, $t$, for Case 1, Case 2 and Case 3, are shown in Figures $6 \mathrm{a}, 6 \mathrm{~b}$ and $6 \mathrm{c}$, respectively. It is apparent from Fig. 6 that the proposed DMP offers higher reward (i.e., throughput) for all $P_{v a c}$ distributions. The decrease in reward in Fig. 6 after first peak is due to CR switching to the RFEH mode after utilizing initial $50 \%$ battery. The gain in reward is lower compared to Fig. 5 since CRs spent substantial amount of time in the RFEH mode due to the higher value of $\theta$ considered in the simulations.

Next, 50 different combinations of $M \in\{3,4, . ., 12\}, B_{v} \preceq(1 / 2)$ and $P_{v a c} \in$ \{Case 1, Case 2, Case 3$\}$ for each of the thirteen distinct parameter settings are considered. The first setting corresponds to the conventional CR mode with no need of RFEH. For the remaining settings, RFEH enabled CRs with limited battery capacity are considered. These twelve settings are divided into two groups of six settings each with $B_{a f e}=3 \cdot B_{c m i n}$ for group 1 and $B_{a f e}=B_{c m i n}$ for group 2. Group 1 takes into account wider contiguous bandwidth for RFEH while group 2 limits the bandwidth to the smallest possible bandwidth i.e., $B_{\text {cmin }}$. Parameters $\left\{\epsilon_{1}, \epsilon_{2}, \theta\right\}$ for these two groups are $\{0.2,0.3,4\},\{0.2,0.3,8\}$, $\{0.2,0.3,15\},\{0.2,0.8,4\},\{0.2,0.8,8\},\{0.2,0.8,15\}$, respectively. Other parameters are same as that of Fig. 6.

In Fig. 7, the comparison is made between different DMPs based on the average reward, $S(t)$ in \% with respect to $S(t)$ of the proposed DMP. For example, if the height of bar is $80 \%$, it means that the reward, $S(t)$, of the corresponding DMP is $80 \%$ of that of the proposed DMP. It can be observed that the proposed DMP outperforms other DMPs in all scenarios. The gain in reward,

$515 S(t)$, is higher when $B_{a f e}=3 \cdot B_{\text {cmin }}$ compared to when $B_{a f e}=B_{c m i n}$. This is due to the proposed Algorithm 4 which identifies the optimum subband of bandwidth, $B_{a f e}$, for the RFEH mode. Lower the value of $\theta$, fewer are the number of time slots for which CRs are in the RFEH mode. As a result, the gain in $S(t)$ increases as $\theta$ decreases as shown in Fig. 7. It is expected that more efficient RFEH circuits with the lower value of $\theta(<4)$ and multi-band harvesting capability $\left(B_{a f e}>B_{c m i n}\right)$ will be available in near future for which the proposed DMP offers more than $15 \%$ gain in $S(t)$ over existing DMPs.

In Fig. 8, the average SSC in \% of different DMPs are compared with respect to that of $\mathrm{UCB}+\rho^{\text {rand }} \mathrm{DMP}$ in [16]. The SSC of the proposed DMP is very low which makes it energy efficient. Similar to Fig. 7, savings in SSC increases as $\theta$ and $B_{a f e}$ increases. In summary, the total reward and SSC of the Bayesian algorithms based DMPs are significantly better than frequentist approach algorithm based DMPs. Also, the proposed DMP not only offers superior performance as shown in Figures 5, 6, 7 and 8 but also has lower computational complexity as discussed in [27-29]. 


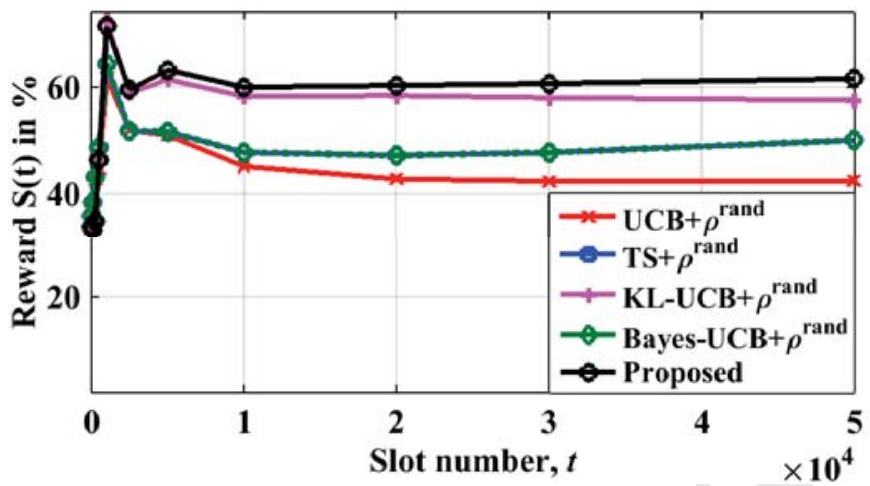

(a)

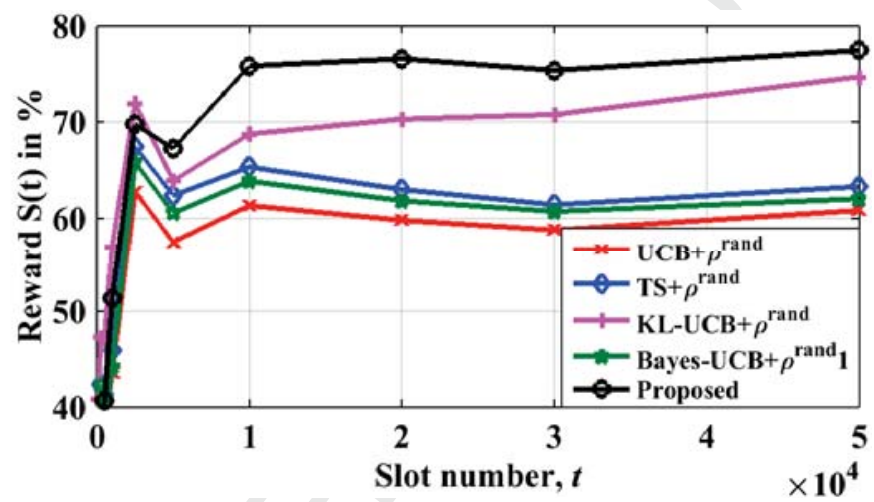

(b)

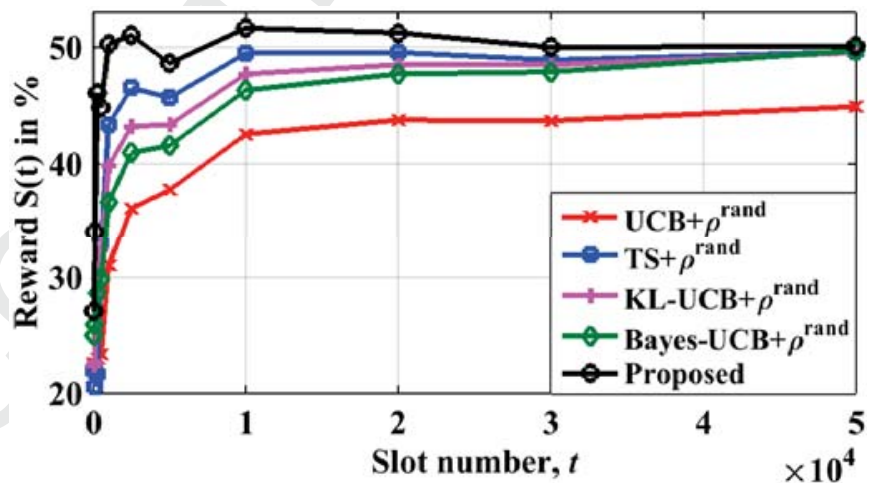

(c)

Figure 6: Average reward, $S(t)$ in $\%$ vs. time slot, $t$, for different $P_{v a c}$ distributions i.e., (a) Case 1, (b) Case 2, and (c) Case 3 for the decentralized CRNs with RFEH enabled CRs having limited battery capacity. 


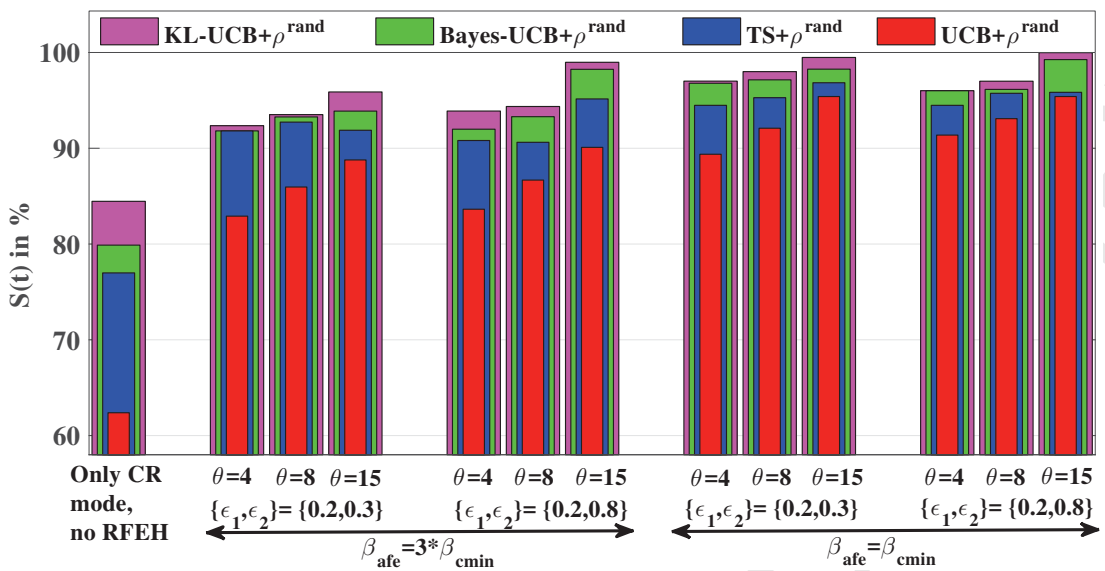

Figure 7: Comparisons of average $S(t)$ in $\%$ of different DMPs with respect to the proposed CR-RFEH DMP.

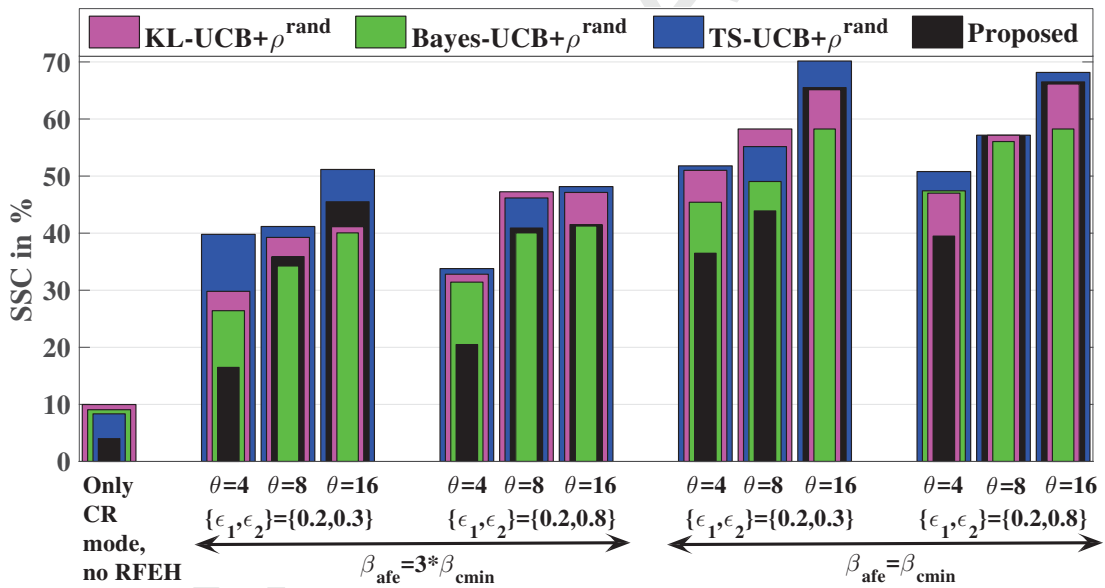

Figure 8: Comparisons of average SSC in \% of different DMPs with respect to the DMP in [16].

\section{Proposed USRP Testbed}

In this section, the functionality of DMPs is validated on the proposed USRP testbed using real radio signals and non-ideal spectrum detector. The proposed testbed, shown in Fig. 9, is an extension of our testbed in [31]. It consists 535 of two units: 1) Left hand side unit is a primary user traffic generator, and 2) Right hand side unit acts as the DMP of CRs. The reader may refer to [31] for implementation details. 


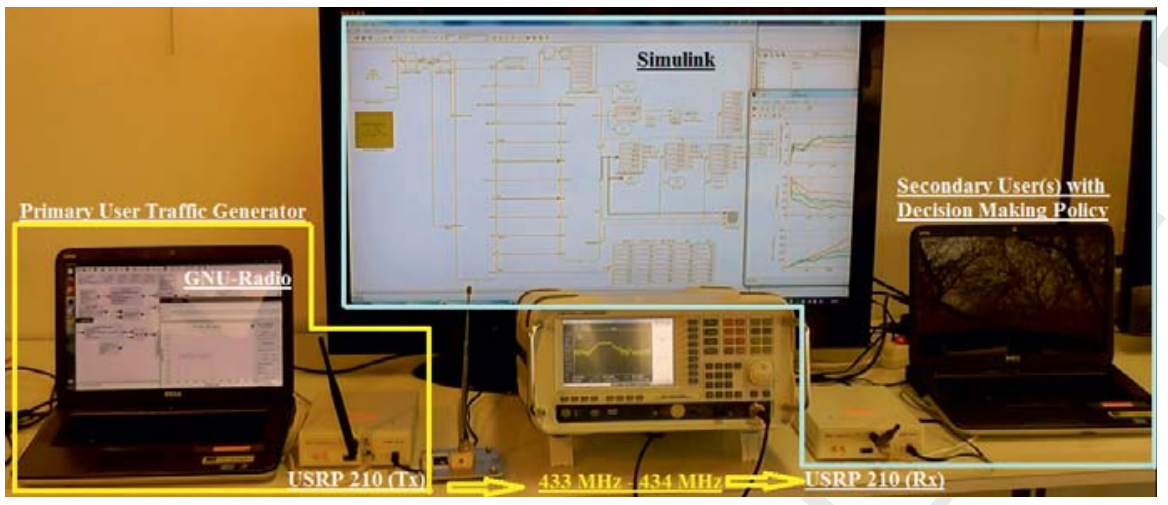

Figure 9: Proposed USRP based testbed for analyzing the performance of DMPs using real radio signals and non-ideal spectrum detectors.

\subsection{Primary User Traffic Generator}

The main task of this unit is to generate the primary user traffic based on the given subband statistics. The chosen design environment for the primary user traffic generation is the GNU Radio Companion (GRC) and the hardware platform is made of the USRP from Ettus Research. The data modulation used is a differential QPSK modulation with Gray encoding and sub-carrier mapping is done using the OFDM. For the experimental results presented in this paper, the number of sub-carriers, center frequency and transmission bandwidth are $256,433.5 \mathrm{MHz}$ and $1 \mathrm{MHz}$, respectively. The center frequency of $433.5 \mathrm{MHz}$ is carefully chosen to avoid the interference to the real licensed users while the transmission bandwidth depends on the analog-front end bandwidth of the USRP. Depending on the number of subbands, $N$, the number of carriers in 550 each subband are calculated. For example, when $N=8$, each subband has 32 carriers. For demonstration purpose, the time slot duration is one second so that it can be followed by human eye. However, it can be reduced to the order of milliseconds and will have no direct effect on the performance of DMPs.

\subsection{Secondary User with Decision Making Policy}

555 The chosen design environment for the CR terminal is Matlab/Simulink and USRP from Ettus Research. The USRP is tuned to receive the signal of bandwidth $1 \mathrm{MHz}$ centered at $433.5 \mathrm{MHz}$. The received signal is then down-sampled, digitized and passed to the DMP implemented using the Matlab/Simulink. The MAB algorithm of the DMP selects one subband in each time slot. The chosen subband is then sensed using the energy detector. Note that the energy detector is not ideal and sensing errors may occur [32]. In the past works, sensing is assumed as perfect which is not true in real radio conditions. Thus, the proposed testbed with non-ideal spectrum detector enables the study of the performance of DMPs in the presence of sensing errors. In case of multiple CRs, each CR 
is independently implemented in the Simulink with their respective DMP. For the RFEH mode, the RF energy of the chosen subband is converted to the corresponding battery capacity as discussed before in Section 3 .

\subsection{Experimental Results}

In this section, we validate the functionality of DMPs on the proposed USRP testbed. Due to the limited memory and delay constraints of USRP and Matlab, the performance comparisons of only two DMPs, UCB $+\rho^{\text {rand }}[16]$ and the proposed DMP are presented. Consider $N=16$ and $B=1 \mathrm{MHz}$, then $B_{c m i n}=62.5 \mathrm{KHz}$. Each numerical result reported hereafter is the average of values obtained over 15 independent experiments on the USRP testbed and each experiment consider a time horizon of 1000 iterations i.e. 1000 time slots for each CR and one time slot corresponds to one second. It is assumed that all CRs employ the same DMP but do not exchange any information with others.

First, CRs with unlimited battery capacity are considered which means that the RFEH is not needed. Fig. 10a shows the average reward (i.e., throughput), $S(t)$, in percentage for two DMPs w.r.t. that of genie-aided DMP. Note that all parameters are same as those considered in Fig. 5. Similarly, CRs with the RFEH capability are considered in Fig. $10 \mathrm{~b}$ and all the parameters are same as those considered in Fig. 6. In Fig. 10a and Fig. 10b, solid lines and dotted lines correspond to Case 1 and Case 2, respectively. It can be observed that the proposed DMP offers 10-15\% higher reward (i.e., throughput) over $\mathrm{UCB}+\rho^{\text {rand }}$ DMP in [16]. Simulation and experimental results on the USRP testbed validate the superiority of the proposed DMP over existing DMPs.

\section{Conclusions}

In this paper, a new decision making policy (DMP) for the opportunistic spectrum access based Cognitive Radios (CRs) with the radio frequency energy harvesting (RFEH) capabilities is proposed. The contributions are the design of Bayesian approach based tunable Thompson sampling algorithm for the CR and RFEH mode, tunable bandwidth subband access scheme using the TS algorithm and the mode selection scheme. Extensive simulations and experimental results

595 using the real radio signal showed the superiority of the proposed DMP over existing DMPs in terms of the throughput of the decentralized network and subband switching cost. Furthermore, lower subband switching cost and lower computational complexity make the proposed DMP suitable for the resourceconstrained battery operated CR terminals.

\section{Acknowledgment}

This work has received the funding support from the department of science and technology (DST), Government of India under the INSPIRE Faculty Fellowship. The authors would like to thank Dr. Emilie Kaufmann, Junior Researcher, CRIStAL, Lille France and Dr. Aditya Gopalan, Assistant Professor, 
605

\section{Sampling algorithm.}

\section{References}

[1] A. Asadi, Q. Wang and V. Mancuso, "A Survey on Device-to-Device Communication in Cellular Networks," accepted in IEEE Communications Surveys and Tutorials, April 2014. http://arxiv.org/abs/1310.0720

[2] P. Phunchongharn, E. Hossain and D. I. Kim, "Resource Allocation for Device-to-Device Communications Underlaying LTE-Advanced Networks," IEEE Wireless Communications, vol. 20, no. 4, pp. 91-100, Aug. 2013.

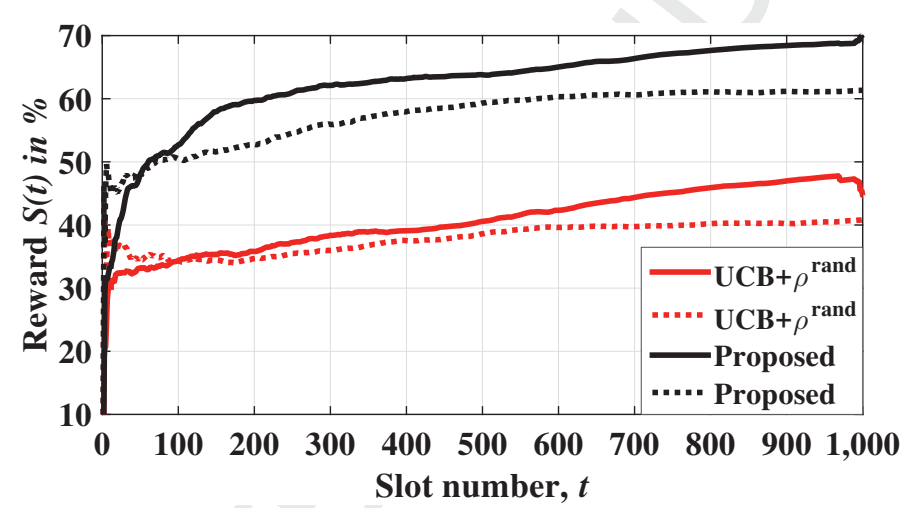

(a)

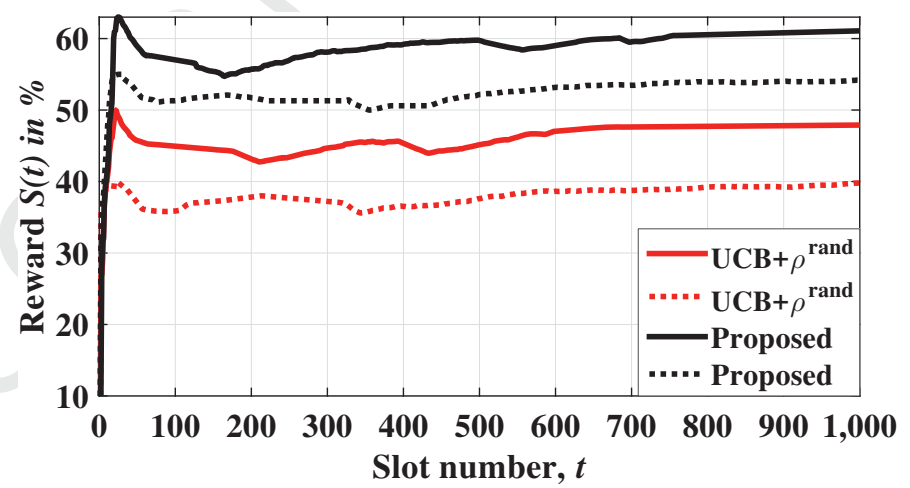

(b)

Figure 10: Average reward, $S(t)$ in $\%$ vs. time slot, $t$, for different $P_{v a c}$ distributions i.e., Case 1 (solid lines), and Case 2 (dotted lines) for the decentralized network consisting of CRs (a) With unlimited battery capacity, i.e. no need of RFEH, (b) With limited battery capacity. 
[3] X. Lu, P. Wang, D. Niyato and E. Hossain, "Dynamic Spectrum Access in Cognitive Radio Networks with RF Energy Harvesting," IEEE Wireless Communications, vol. 21, no. 3, pp. 102-110, June 2014.

[4] G. Zheng, Z. Ho, E. A. Jorswieck and B. Ottersten, "Information and Energy Cooperation in Cognitive Radio Networks," IEEE Transactions on Signal Processing, vol. 62, no. 9, pp. 2290-2303, May 2014.

[5] S. Park, J. Heo, B. Kim, W. Chung, H. Wang and D. Hong, "Optimal Mode Selection for Cognitive Radio Sensor Networks with RF Energy Harvesting," in Proc. IEEE $23^{\text {rd }}$ International Symposium on Personal, Indoor and Mobile Radio Communications (PIMRC), Sydney, Australia, Sept. 2012.

[6] X. Lu, P. Wang, D. Niyato, D. I. Kim and Z. Han, "Wireless Networks with RF Energy Harvesting: A Contemporary Survey," arXiv preprint arXiv:1406.6470, June 2014.

[7] D. T. Hoang, D. Niyato, P. Wang and D. I. Kim, "Opportunistic Channel Access and RF Energy Harvesting in Cognitive Radio Networks," IEEE Transactions on Selected Areas in Communications, vol. 32, no. 11, pp. 114, Nov. 2014.

[8] P. Nintanavongsa, U. Muncuk, and D. R. Lewis, "Design Optimization and Implementation for RF Energy Harvesting Circuits ," IEEE Journal on Emergin and Selected Topics in Circuits and Systems, vol. 2, no. 1, pp. 24-33, Mar. 2012.

[9] L. Mohjazi, M. Dianati, G. Karagiannidis, S. Muhaidat and M. Al-Qutayri, "RF-Powered Cognitive Radio Networks: Technical Challenges and Limitations ," IEEE Communication Magazine, pp. 94-100, April 2015.

[10] X. Hong, J. Wang, C-X. Wang and J. Shi, "Cognitive Radio in 5G: A Perspective on Energy-Spectral Efficiency Trade-off," in IEEE Communication Magazine, vol. 52, no. 7, July 2014.

[11] C. Herranz, V. Osa, J. F. Monserrat, D. Calabuig, N. Cardona and X. Gelabert, "Cognitive Radio Enabling Opportunistic Spectrum Access in LTE-Advanced Femtocells," in Proc. IEEE International Conference on Communications (ICC'12), pp. 5593-5597, Ottawa, ON, June 2012.

[12] Z. Qin, Y. Liu, Y. Gao, M. Elkashlan and A. Nallanathan, "Throughput Analysis for Compressive Spectrum Sensing with Wireless Power Transfer," in Proc. IEEE Global Telecommunications Conference (GLOBECOM'15), San Diego, USA, Dec. 2015.

[13] S. J. Darak, H. Zhang, J. Palicot and C. Moy, "Compute-Efficient Decision Making Policy for D2D Communications and RF Energy Harvesting in Cognitive Radio Networks: Go Bayesian!," accepted in $23^{\text {rd }}$ European Signal Processing Conference (EUSIPCO), , Nice, France, Aug. 2015. 
[14] Y. Xu, A. Anpalagan, Q. Wu, L. Shen, Z. Gao and J. Wang, "DecisionTheoretic Distributed Channel Selection for Opportunistic Spectrum Access: Strategies, Challenges and Solutions," IEEE Communications Surveys and Tutorials, vol. 15, no. 4, pp. 1689-1713, Nov. 2013.

[15] P. Auer, N. Cesa-Bianchi and P. Fischer, "Finite-time Analysis of the Multiarmed Bandit Problem," Machine Learning, vol. 47, no. 2, pp. 235256, 2002.

[16] A. Anandkumar, N. Michael, A. Tang and A. Swami, "Distributed Algorithms for Learning and Cognitive Medium Access With Logarithmic Regret," IEEE Journal on Selected Areas in Communications, vol. 29, no. 4, pp. 731-745, April 2011.

[17] K. Liu and Q. Zhao, "Distributed Learning in Multi-Armed Bandit with Multiple Players," IEEE Transactions on Signal Processing, vol. 58, no. 11, pp. 5667-5681, Nov. 2010.

[18] M. Zandi and M. Dong, "Learning-Stage Based Decentralized Adaptive Access Policy For Dynamic Spectrum Access," in IEEE International Conference on Acoustics, Speech and Signal Processing (ICASSP), Vancouver, Canada, May 2013.

[19] M. Zandi, M. Dong and A. Grami, "Decentralized spectrum learning and access adaptive to channel availability distribution in primary network," in Proc. $14^{\text {th }}$ Workshop on Signal Processing Advances in Wireless Communications (SPAWC), Darmstadt, Germany, June 2013.

[20] S. J. Darak, H. Zhang, J. Palicot and C. Moy, "Efficient Decentralized Dynamic Spectrum Learning and Access Scheme for Multi-standard Multi-user Cognitive Radio Networks," accepted in $11^{\text {th }}$ International Symposium on Wireless Communication Systems (IEEE ISWCS'2014), Barcelona, Spain, Aug. 2014.

[21] Z. Qin, Y. Gao, M. Plumbley and C. Parini, "Wideband Spectrum Sensing on Real-time Signals at Sub-Nyquist Sampling Rates in Single and Cooperative Multiple Nodes," IEEE Transactions on Signal Processing, vol. 64, no. 12 , pp. 3106-3117, June 2016.

[22] A. Ambede, K. G. Smitha, and A. P. Vinod, "A Low Complexity Uniform and Non-Uniform Digital Filter Bank based on an Improved Coefficient Decimation Method for Multi-Standard Communication Channelizers," Circuits, Systems and Signal Processing, Springer, vol. 32, no. 6, pp. 2543-2557, Dec. 2013.

${ }_{690}$ [23] P. Löwenborg and H. Johansson, "Minimax Design of Adjustable Bandwidth Linear-Phase FIR Filters," IEEE Transactions on Circuits and Systems-I, vol. 53, no. 2, pp. 431-439, Feb. 2006. 
[24] S. J. Darak, A. P. Vinod, E. M-K. Lai, J. Palicot, and H. Zhang, "Linear Phase VDF Design with Unabridged Bandwidth Control over the Nyquist Band," IEEE Transactions on Circuits and Systems-II, vol. 61, no. 6, pp. 428-432, Apr. 2014

[25] S. J. Darak, A. P. Vinod, and E. M-K. Lai, "Efficient Implementation of Reconfigurable Warped Digital Filters with Variable Lowpass, Highpass, Bandpass and Bandstop Responses," IEEE Transactions on Very Large Scale Integration Systems, vol. 21, no. 6, pp. 1165-1169, July 2012.

[26] S. J. Darak, S. Dhabhu, C. Moy, H. Zhang, J. Palicot and A. P. Vinod, "Low Complexity and Efficient Dynamic Spectrum Learning and Tunable Bandwidth Access for Heterogeneous Decentralized Cognitive Radio Networks," Digital Signal Processing (Elsevier), vol. 37, pp. 13-23, Feb. 2015.

[27] E. Kaufmann, N. Korda and R. Munos, "Thompson Sampling: An Asymptotically Optimal Finite Time Analysis," Algorithmic Learning Theory, pp. 199-213, Oct. 2012.

[28] S. Agrawal and N. Goyal, "Further Optimal Regret Bounds for Thompson Sampling," in Proc. $16^{\text {th }}$ International Conference on Artificial Intelligence and Statistics (AISTATS), Scottsdale, USA, April 2013.

[29] D. Russo and B. V. Roy, "An Information-Theoretic Analysis of Thompson Sampling, " submitted to Computing Research Repository (CoRR), Mar. 2014. http://arxiv.org/abs/1403.5341

[30] http://www.powercastco.com/documentation/

715 [31] S. J. Darak, A. Nafkha, C. Moy and J. Palicot, "Is Bayesian Multi-armed Bandit Algorithm Superior?: Proof-of-Concept for Opportunistic Spectrum Access in Decentralized Networks," accepted in $11^{\text {th }}$ International Conference on Cognitive Radio Oriented Wireless Networks (CROWNCOM), Grenoble, France, May 2016.

${ }_{720}[32]$ S. Bahamou and A. Nafkha, "Noise Uncertainty Analysis of Energy Detector: Bounded and Unbounded Approximation Relationship," in $21^{\text {st }} \mathrm{Eu}$ ropean Signal Processing Conference (EUSIPCO), pp. 1-4, Marrakech, Morocco, Sept. 2013.

Sumit J. Darak received his B.E. degree in Electronics and Telecommunications Engineering Pune University, India in 2007, and PhD degree from the School of Computer Engineering, Nanyang Technological University (NTU), Singapore in 2013. He is currently an Assistant Professor at Indraprastha Institute of Information Technology, Delhi (IIITD), India. Prior to that, he was working as Assistant System Engineer in Tata Consultancy Services (TCS), Pune, India from September 2007 to December 2008. From March 2013 to November 2014, he was 730 pursuing postdoctoral research at the CominLabs Excellence Center, Université Europèenne 
de Bretagne (UEB) and Supélec, Rennes, France. Dr. Sumit has been awarded India Government's "DST Inspire Faculty Award" which is a prestigious award for young researchers under 32 years age. His current research interests include non-uniform sampling, design of area and power efficient variable digital filters and reconfigurable filter banks for multi-standard wire-

735 less communication transceivers, decision making and learning algorithms for various wireless communication applications such as D2D communications and RF energy harvesting etc.

Honggang Zhang is a Full Professor of Department of Information Science and Electronic Engineering as well as the Co-Director of York-Zhejiang Lab for Cognitive Radio and Green Communications at the Zhejiang University, China. He is an Honorary Visiting Professor of the University of York, UK. He received the Ph.D. degree in Electrical Engineering from Kagoshima University, Japan, in March 1999. From October 1999 to March 2002, he was with the Telecommunications Advancement Organization (TAO) of Japan, as a TAO Research Fellow. From April 2002 to November 2002, he joined the TOYOTA IT Centre. From December 2002 to August 2004, he has been with the UWB Research Consortium, Communications Research Laboratory (CRL) and National Institute of Information and Communications Technology (NICT) of Japan. He was the principle contributor for proposing DS-UWB in IEEE 802.15 WPAN standardization task group. From September 2004 to February 2008, he has been with CREATE-NET (Italy), where he leaded its wireless team in exploring Cognitive 750 Radio and UWB technologies while participated the European FP6/FP7 projects (EUWB, PULSERS2). He was an International Chair Professor, CominLabs Excellence Center, Université Europèenne de Bretagne (UEB) \& Supélec, France from January 2013 till November 2014. Dr. Honggang Zhang has served as the Chair of the Technical Committee on Cognitive Networks (TCCN) of the IEEE Communications Society (ComSoc) during 2011-2012. He is the Co-Chair of IEEE Globecom 2008 Symposium and IEEE ICC 2013 Symposium. He was the founding TPC Co-Chair of CrownCom 2006 and the Steering Committee Member of CrownCom 2006-2009. In the area of green communications and networks, Dr. Honggang Zhang was the Lead Guest Editor of the IEEE Communications Magazine feature topic issues on "Green Communications". He is the General Co-Chair of IEEE GreenCom 2010 (2010 IEEE nternational Conference on Green Computing and Communications) and the TPC Co-Chair of IEEE OnlineGreenComm 2014 (The 2014 IEEE Online Conference on Green Communications). He is the Series Editor of IEEE Communications Magazine (Green Communications and Computing Networks Series). He is the co-editor/co-author of two books with the titles of "Cognitive Communications - Distributed Artificial Intelligence (DAI), Regulatory Policy

765 \& Economics, Implementation" (Wiley Press) and "Green Communications: Theoretical Fundamentals, Algorithms and Applications"(CRC Press), respectively.

Prof. Jacques Palicot received, in 1983, his PhD degree in Signal Processing from the University of Rennes. Since 1988, he has been involved in studies about equalization techniques applied to digital transmissions and analog TV systems. Since 1991, he has been involved mainly in studies concerning the digital communications area and automatic measurements techniques. He has taken an active part in various international bodies EBU, CCIR, URSI, and within RACE, ACTS and IST European projects. He has published various scientific articles notably on equalization techniques, echo cancellation, hierarchical modulations and Software Radio techniques. He is author or co-author of more than 300 publications with more than 50 in prestigious journals, two books and 22 patents. He is currently involved 
in adaptive Signal Processing, digital communications, Software Radio, Cognitive radio and Green Radio. From November 2001 to September 2003, he had a temporary position with INRIA/IRISA in Rennes. He serves as Associate Editor for EURASIP JASP since 2008. He also served as lead guest editor for several Special Issues on Software Radio, Cognitive Radio and Green Radio. He was Co-General Chairman of ISCIT 2011, Co-General Chairman of Next-GWiN 2014, Technical Program Chairman of CROWNCOM 2009, Technical Program Chairman of GREENCOM 2013 and Technical Program Chairman of CRN Symposium of ICC 2014. Since October 2003, he is with CentraleSupélec in Rennes where he leads the

Signal, Communications and Embedded Electronics (SCEE) research team.

Christophe Moy received the engineer diploma of the National Institute of Applied Sciences (INSA), Rennes, France, in 1995. He received the M.Sc. and Ph.D. degrees in electronics in 1995 and 1999, respectively, from the INSA. He then worked 6 years at Mitsubishi Elec-

790 tric ITE-TCL research lab where he was focusing on Software Radio systems and concepts, including digital signal processing, HW and SW architecture, co-design methodology and reconfiguration management. He represented Mitsubishi Electric at the SDR Forum and worked on French research program A3S, and IST European project $E^{2} R$. Since 2005, he has been working as a Professor in Supélec, now CentraleSupélec since January 2015, in IETR entity of CNRS (UMR 6164): Institue of Electronics and Telecommunications of Rennes. His research focuses on Software Radio, Cognitive Radio and Green Radio. He addresses heterogeneous design techniques for SDR, high-level design methodologies for cognitive management, decentralized decision making and learning for cognitive radio equipments and systems, dynamic spectrum access. He was involved in several European collaborative projects: Networks of Excellence NEWCOM and NEWCOM++, EULER, $E^{2} R$-phase2. He participated also to several French collaborative projects (ANR): Mopcom and Idromel. He currently is the head of the Communication Department of IETR. He is working in the following collaborative projects: French ANR WiNoCoD and SoGreen, "pole Image \& Reseaux" SoftRF, CominLabs labex TEPN. He is also a member of Institute of Research and Technology B-COM, in Rennes. He participates to ETSI RRS standardization for B-COM. Christophe MOY has written more than 20 book chapters, more than 20 journal papers and around 100 conference papers. 